\title{
Ambient Pressure Photoelectron Spectroscopy: A new tool for surface science and nanotechnology
}

\author{
M. Salmeron ${ }^{1}$ and R. Schlögl ${ }^{2}$ \\ ${ }^{1}$ Lawrence Berkeley National Laboratory and \\ Materials Science and Engineering Department, University of California, Berkeley \\ ${ }^{2}$ Fritz-Haber Institute of the Max Planck Gesellshaft. Berlin. Germany
}




\section{$\underline{\text { Table of contents }}$}

\section{Introduction}

\section{Techniques to study surfaces under ambient pressure conditions}

\section{Problems and solutions in electron-based spectroscopies}

3.1 Electron mean free path and differential pumping

\subsection{History}

3.3 The New Ambient Pressure Photo-Electron Spectrometer

3.3.1 Transfer electron optics

3.3.2 Analysis and preparation chambers

3.3.3 X-ray windows

\section{X-ray electron spectroscopies}

\subsection{XPS and XAS}

4.2 Calibration and background signal issues

4.3 Charging and beam damage effects

4.4 Gas phase spectra

\section{Applications to environmental science}

5.1 The melting of ice

5.2 The surface of aqueous ionic solutions

5.3 Water films on surfaces

5.3.1 Water on $\mathrm{TiO}_{2}(110)$

5.3.2 Water on amorphous $\mathrm{SiO}_{2}$

6. Applications to catalysis

6.1 Oxidation of Pd(111)

6.2 Redox Chemistry of Cu: Oxidation of $\mathrm{Cu}_{2} \mathrm{O}$ to $\mathrm{CuO}$

6.3 Reduction of a Cu/ZnO nanostructured system

6.4 Catalytic reactions

6.4.1 Copper in $\mathrm{MeOH}$ oxidation

6.4.2 Ruthenium: oxidation of $\mathrm{CO}$ and $\mathrm{MeOH}$ 
6.4.3 Copper in $\mathrm{MeOH}$ steam reforming

6.4.4 Automobile catalysis: coadsorption of $\mathrm{CO}$ and $\mathrm{NO}$ on $\mathrm{Rh}$

6.4.5 Poisoning of $\mathrm{H}_{2}$ chemisorption by $\mathrm{CO}$ on Pt(111)

\section{Conclusions and outlook}

\section{Acknowledgments}




\section{Introduction}

Progress in science often follows or parallels the development of new techniques. The optical microscope helped convert medicine and biology from a speculative activity in old times to today's sophisticated scientific disciplines. The telescope changed the study and interpretation of heavens from mythology to science. X-ray diffraction enabled the flourishing of solid state physics and materials science. The technique object of this review, Ambient Pressure Photoelectron Spectroscopy or APPES for short, has also the potential of producing dramatic changes in the study of liquid and solid surfaces, particularly in areas such as atmospheric, environment and catalysis sciences. APPES adds an important missing element to the host of techniques that give fundamental information, i.e., spectroscopy and microscopy, about surfaces in the presence of gases and vapors, as encountered in industrial catalysis and atmospheric environments. APPES brings electron spectroscopy into the realm of techniques that can be used in practical environments. Decades of surface science in ultra high vacuum (UHV) has shown the power of electron spectroscopy in its various manifestations. Their unique property is the extremely short elastic mean free path of electrons as they travel through condensed matter, of the order of a few atomic distances in the energy range from a few $\mathrm{eV}$ to a few thousand $\mathrm{eV}$. As a consequence of this the information obtained by analyzing electrons emitted or scattered from a surface refers to the top first few atomic layers, which is what surface science is all about. Low energy electron diffraction (LEED), Auger electron spectroscopy (AES), X-ray photoelectron spectroscopy (XPS), Ultraviolet photoelectron spectroscopy (UPS), and other such techniques have been used for decades and provided some of the most fundamental knowledge about surface crystallography, composition and electronic structure available today. Unfortunately the high interaction cross section of electrons with matter also prevents them from traveling long distances unscattered in gas environments. Above the millibar pressure range this distance is reduced to less that a millimeter, effectively preventing its use in the most relevant environments, usually between millibars and atmospheric pressures. There is therefore a large gap of several orders of magnitude where information about surfaces is scarce because these powerful electron spectroscopies cannot operate. 
One characteristic of surfaces in ambient pressure environments is that they are covered by dense layers of molecules, even when their binding energy is weak. Water for example is known to form layers several molecules thick at room temperature in humid environments. Metals readily form oxide films several layers thick in oxygen atmospheres. Dense layers of adsorbed molecules can also be produced in ultra high vacuum, often by the simple and expedient method of cooling the sample to cryogenic temperatures. A large amount of data has been obtained in the past in UHV by surface scientists using this method. While this has provided valuable information it begs the question of whether the structures formed in this manner represent equilibrium structures or metastable ones, kinetically trapped due to high activation energies that cannot be overcome at low temperature.

From a thermodynamic point of view is interesting to consider the entropic contribution to the Gibbs free energy, which we can call "the pressure factor", equal to kT. $\log$ P. This factor amounts to a sizeable $0.3 \mathrm{eV}$ difference at room temperature between UHV ( $<10^{-8}$ Pascal) and atmospheric pressures. Such change if free energy can definitely result in changes in surface structure and stability. Entire areas of the phase diagram are out of reach due to the pressure gap.

Even when cooling is not necessary, many surface treatments and most chemical reactions necessitate the presence of gases at pressures ranging from millibar to bars. What is the structure and chemical nature of the species formed on the surface in equilibrium with such gases? As we shall illustrate in this review, APPES provides a much needed electron spectroscopy to analyze surface electronic structure and composition in equilibrium with gases.

The review is organized as follows. After a brief general discussion of techniques for in situ studies of surfaces under ambient conditions (Section 2), section 3 describes the technical details of the operation and implementation of APPES and how to circumvent the problem of electrons traveling through gas phases. This section includes also a brief summary of previous attempts to implement in situ photoelectron spectroscopy. Section 4 deals with the two most widely used photoelectron techniques, XPS and XAS and the specific problems encountered in ambient gas pressure 
environments. Following these technical sections, we present a summary of results obtained by application of APPES in the three currently operating instruments, two at the Advanced Light Source in Berkeley (ALS), and one at BESSY in Berlin. Section 5 is devoted to environmental studies, mostly dealing with water vapor environments and section 6 to catalysis studies. A conclusion and future outlook concludes the review.

\section{Techniques to study surfaces under ambient pressure conditions}

Techniques that provide spectroscopic and microscopic information of surfaces while exposed to gases or liquids in the atmospheric pressure range are highly desirable for fundamental studies of catalysis and environmental science. Any technique is based in the response of a material to an external excitation. The excitation is accomplished by probe particles (electrons, atoms, ions) or by radiation (photons, heat, magnetic fields), the response consisting in the emission of particles or in the absorption of the probe. Strong absorption or scattering of the probing or emitted particles forbids or makes difficult experiments where the sample is inside of a dense media, due to the difficulty of separating processes that took place in the sample from those that occurred in the surrounding medium. This puts severe restrictions in the type or probe or emitted particle that can be used.

Probes that interact weakly with the media are natural candidates for studies of surfaces under ambient pressures. These include radiation from various parts of the electromagnetic spectrum, from microwaves to x-rays. The repertoire of techniques traditionally used in gas environments include Thermal Reaction Spectroscopy (TRS), Nuclear Magnetic Resonance (NMR), Electron Spin Resonance (ESR), and optical techniques based on photon-in and photon-out processes, like Infrared Absorption (IR), Ellipsometry, Raman, Sum Frequency Generation, x-ray absorption and emission spectroscopy (XAS, XES), x-ray diffraction, etc. Weakly interacting particles such as neutrons can also be used. Because of their weak interaction however, many of these probes are not very sensitive to the surface due their large penetration depths inside the material. Surface sensitivity can be obtained when the species at the surface are different 
from those in the bulk and thus contribute uniquely to the excitation or absorption spectra.

It is from techniques that utilize electrons, ions and atoms that some of the most detailed and fundamental information about surfaces has been obtained. The field of modern Surface Science was developed around techniques such as Transmission Electron Microscopy (TEM), Scanning Electron Microscopy (SEM), Low Energy Electron Diffraction (LEED), Auger Spectroscopy, X-ray Photoelectron Spectroscopy (XPS), Ion Scattering, and electron yield x-ray absorption. Electrons and ions in the energy range of a few hundred $\mathrm{eV}$ are strongly absorbed or scattered and therefore do not penetrate into the material except for a very few number of atomic layers. The information carried out by emitted or scattered particles is therefore highly surface sensitive.

Due to the strong interaction of electrons and ions with the gas, these surface science techniques have been used almost exclusively in high vacuum environments, below $10^{-4}$ $\mathrm{Pa}\left(=10^{-9}\right.$ bar $)$. Several methods to circumvent this problem have been developed. They all involve capturing the particles at distances comparable with their mean free path, which for electrons of a few hundred $\mathrm{eV}$ at millibar type pressures is in the millimeter range. This is accomplished by means of small apertures near the sample and by differential pumping to remove gases leaking through it.

Although not part of the electron-based spectroscopies reviewed here, it is also appropriate to mention another class of probes based also on proximity of source and detector. These are Scanning Tunneling and Atomic Force Microscopy, also known collectively as Scanning Probe Microscopies (SPM). They have made a huge impact in traditional surface science and in air, gas and liquid environments. In SPM an atomically sharp tip is brought in contact with the sample, or within nanometer distances. Electron tunneling, which samples electronic and vibrational levels, and interaction forces of attractive and repulsive character (van der Waals, bonding, electrostatic, etc.) are used to image the surface with atomic resolution. Numerous articles have been published on the applications of high pressure SPM to catalysis science and we refer ${ }^{[1,2]}$ the reader to these for further information. 


\section{Problems and solutions in electron-based spectroscopies}

\subsection{Electron mean free path and differential pumping}

As the electrons emitted by the sample travel through the gas phase they experience elastic and inelastic collisions with molecules. The mean free path $\lambda_{\mathrm{e}}$, or average distance between collisions depends on their energy and gas pressure P. Figure 1 shown the variation of $\lambda_{\mathrm{e}}$ as a function of electron energy in an oxygen gas environment. As can be seen, for electrons of $400 \mathrm{eV}$ kinetic energy $\lambda_{\mathrm{e}}$ is about $4 \mathrm{~mm}$ when the pressure is 1 mbar. Similar values are found for other gases. Since $\lambda_{\mathrm{e}}$ is inversely proportional to $\mathrm{P}$, its value decreases to about $30 \mu \mathrm{m}$ at $100 \mathrm{mbar}$, which makes collection of unscattered electrons very challenging. As a result of scattering the count rate of detected electrons decreases exponentially with distance, indicating the need to shorten the travel distance in the high pressure region as much as possible. All instruments developed to make possible electron spectroscopy under environmental gases are based on this idea, which is accomplished by bringing the sample close to a small aperture at a distance equal or less than $\lambda_{\mathrm{e}}$. Differential pumping is the key to lower the pressure at the other side of the aperture to decrease the collision rate. It is therefore appropriate to briefly review ${ }^{[3]}$ here the conditions of gas flow and pressure distribution in the geometry of these instruments.

Collisions between gas molecules are also characterized by a mean free path $\lambda_{\mathrm{g}}$, which around room temperature and $1 \mathrm{mbar}$ is about $0.05 \mathrm{~mm}$ for $\mathrm{O}_{2}$ and $\mathrm{N}_{2}$. Since the radius of the aperture $\mathrm{R}$ and sample distance $\mathrm{d}$, are in the millimeter range, the Knudsen number $\lambda_{\mathrm{g}} / \mathrm{d}$ is about 0.05 , indicating gas-flow regime above $\mathrm{P}_{1}=1 \mathrm{mbar}$ and molecular flow below $10^{-5}$ bar. When the pressure in the chamber connected to the aperture $\mathrm{P}_{2}$ is lower than $\mathrm{P}_{1} / 2$, which is the usual case, the flow of gas is independent of $\mathrm{P}_{2}$ and has a limiting value of approximately $65 \mathrm{P}_{1} \mathrm{~A}$ Pa-liter/s in the glass-flow regime $\left(\mathrm{P}_{1}>1 \mathrm{mbar}\right)$, where $\mathrm{A}\left(=\pi \cdot \mathrm{R}^{2}\right)$ is the area of the aperture in $\mathrm{mm}^{2}$. For $\mathrm{R}=0.5 \mathrm{~mm}$ the flow rate through the orifice in the gas-flow regime is $15 \mathrm{~Pa}-\mathrm{L} / \mathrm{s}$. When $\mathrm{P}_{1}<10^{-5}$ bar the flow is in the molecular regime, which is controlled by collision with the walls, and the flow rate is given by the molecular flux rate $\mathrm{P}_{1} /(2 \pi \mathrm{mkT})^{1 / 2}$ per unit area. This expression gives a 
value only slightly different from that obtained using the viscous flow expression at 1 mbar.

The Reynolds number of the gas flowing through $1 \mathrm{~mm}$ diameter orifice at 1 mbar is close to 15 , which indicates that the flow regime is viscous or laminar. Because of the geometry of the streamlines funneled through the aperture into the pumping chamber, the gas pressure distribution is not uniform around the sample. Although an exact solution of the flow distribution can be obtained with the help of appropriate software programs, we can already draw a few conclusions regarding this distribution. For this consider an electron traveling along the axis perpendicular to the aperture plane at position $\mathrm{z}$, with $\mathrm{z}$ measured in units of $\mathrm{R}$ from the center of the aperture, with positive $\mathrm{z}$ on the vacuum side. The effective pressure at $\mathrm{z}$ is proportional to the included solid angle in the high-

pressure region, so $\mathrm{P}(\mathrm{z})=1 / 2 \cdot \mathrm{P}_{1}\left(1-\mathrm{z} /\left(1+\mathrm{z}^{2}\right)\right.$ ) (assuming that $\mathrm{P}_{2}$ is negligible compared to $\mathrm{P}_{1}$ ). Although close to the aperture the pressure at the sample surface is less than $\mathrm{P}_{1}$, it increases quickly away from it. At $\mathrm{z}=-2 \mathrm{R}$ the pressure is already $95 \%$ of $\mathrm{P}_{1}$, while at $\mathrm{z}=$ $+2 \mathrm{R}$ it is only $5 \%$. In many of the experiments described below $\mathrm{d} \sim 0.5-2 \mathrm{~mm}$ and $\mathrm{R}=$ $0.25 \mathrm{~mm}$, so that the pressure near the sample is very close to $P_{1}$.

\subsection{History}

The history of ambient pressure XPS dates back to the beginning of the method, when electron spectroscopy was used to study the electronic structure of molecules. A school of instrumentation evolved around the study gaseous organic molecules ${ }^{[4]}$ using He I and He II excitation sources. Since no windows were used in these instruments the sample pressure was about equal to that of the gas discharge ${ }^{[5]}$, which ranged up to several mbar.

Core level electron spectroscopy was performed from the very beginning on gases ${ }^{[5,}$ ${ }^{6]}$ and liquids ${ }^{[7,8]}$, providing fundamental information of chemical effects on the atomic structure. This was due to the intrinsically higher resolution of gas phase spectra as compared to solid samples. The phenomenon of a substantial shift of the binding energy of even simple gases like nitrogen with increasing pressure (up to $2 \mathrm{eV}$ ) was noted. These fundamental studies also revealed clearly that gases and liquids of the same organic molecule may exhibit substantial "chemical" shifts in binding energy pointing to different 
structures in the two aggregate states caused by intermolecular ${ }^{[9]}$ interactions. Designs $^{[10]}$ with magnetic and electrostatic energy analyzers and a versatile sample environment for gaseous and liquid samples were realized. A particularly useful design ${ }^{[11]}$ allowing for angular-resolved studies of solutions was used to derive fundamental aspects of electric double layers in solutions. The first application of high pressure photoemission in gassolid reactivity ${ }^{[12]}$ was made in the United Kingdom in a study of the interaction of oxygen ${ }^{[13]}$ with coin metals. The design involved differential pumping and was capable to operate at pressures up to 1 mbar. Several versions of this design were commercialized and are in operation until today. Their substantial disadvantage was the contamination of the sample by the pumping system based on oil diffusion pumps. In these pioneering studies the effect of adsorbents in enhancing dissolution and compound formation was clearly recognized.

These developments and the valuable information they provided for catalytic studies and in the emerging field of interface science led to the design of an improved version ${ }^{[14]}$ that avoids sample contamination. All these systems were of a "passive" design in the sense that the emitted photoelectrons were simply admitted and spatially filtered through apertures separating the sample in high-pressure environment from the detection chamber at lower pressure. This was changed with a new design ${ }^{[15]}$, developed for the first time at the Advanced Light Source (ALS) in the Berkeley Laboratory, where an electrostatic lens system was used to focus the photoelectrons emitted through an aperture. This and a second generation developed soon after will be discussed in the next section of this review. This novel instrumentation made possible the investigation of fluid and solid samples at higher pressures than ever before. Implementation of the technique required also the use of X-ray windows to protect the UHV of the storage ring from possible pressurization.

A laboratory version of an electrostatic analyzer with differential pumping and electrostatic focusing was developed also in other laboratories, coupled to a molecular beam ${ }^{[16]}$ dosing system. In this way the background pressure seen by the X-rays and the photoelectrons can be substantially reduced without sacrificing a high pressure at the spot of the sample being under study. The study of thin film systems initiated by Grunze et al. 
was carried further and a new dedicated ${ }^{[17]}$ high pressure instrument was built and used for thin film processing including reactive sputtering and chemical vapor deposition.

Although high-pressure photoemission is clearly an important development it had until now found comparatively little application. The scarce availability of laboratory instruments, the longstanding problems of sample contamination and limited data quality have been important obstacles responsible for this retarded development. With the advent of user end stations in several synchrotron sources in the world and the increased availability of commercial laboratory instruments it can be expected that the method will become more popular than in the past two decades. Fundamental contributions to the synthesis, dynamics and chemical reactivity of thin film and nanostructured systems should result from a widespread application of the methodology having shown its potential already in several areas of application.

\subsection{The New Ambient Pressure Photo-Electron Spectrometer}

As mentioned above, the key feature of the new design approach is the addition of electrostatic lens focusing into the differential pumping scheme. This makes possible the collection a substantially larger fraction of electrons that would otherwise be lost due to the diverging nature of their trajectories and the small solid angle captured by the apertures separating the pumping stages. In our design these apertures are located at the focal or crossover points of the electrostatic lenses, as schematically illustrated in the diagrams of figure 2. Two generations of instruments using electrostatic focusing in combination with differential pumping have been developed so far. The first one, located in beamline 9.3.2 of the Berkeley ALS, was developed ${ }^{[15]}$ in 1999-2001. A second generation with improved electron optics and a new chamber design for easier sample manipulation and transfer was developed in 2003-2004. Two versions of this new instrument are currently operating, one is located at beamline 11.0.2 of the ALS and the other at beamline U49/2-PGM1 at BESSY, in Berlin, Germany. The basic design details of the new generation of HPPES are described below. 


\subsubsection{Transfer electron optics}

The transfer optics is a crucial part of the APPES design. The first aperture, or nozzle, is shaped conically with a half angle of $35^{\circ}$. This cone is detachable and can be exchanged by similar cones of different aperture diameters. Figure 3 shows a side and top view of a nozzle. After the nozzle, two sets of Einzel lenses provide focusing into the aperture of a second differentially pumped chamber. Another lens in this chamber refocuses the beam into the third chamber, where a quadrupole lens steers the beam to the entrance of a commercial Phoibos (SPECS) hemispherical analyzer modified to couple with the focusing lens system. The first two chambers are pumped by turbomolecular pumps while the last one, containing the analyzer, is pumped by an ion pump. The lenses are surrounded by $\mu$-metal for magnetic shielding. A schematic diagram of the lens and pumping stages, together with an electron trajectory through the lens system is shown in figure 4(a) and (b). An artistic rendition of the overall system is shown in (c). In this drawing the replaceable nozzle is shown in green, the lens system in gray and the $\mu$-metal in red. The hemispherical analyzer is in yellow.

\subsubsection{Analysis and preparation chambers}

The system is designed with two chambers separated by a gate valve. Additionally a load-lock allows for easy introduction of samples in the chambers with minimal perturbation to the base pressure. The preparation chamber can be optionally equipped with surface science instruments, including cleavage devices, high temperature heating, ion sputtering, Auger Spectroscopy, LEED, etc. After preparation the sample is moved to the reaction chamber using transfer rods and pressurized with the desired gases. The manipulator and sample holder in this high pressure chamber need to be carefully designed since heating in the presence of gases at pressures of several mbar requires special materials. In particular, reactive materials that might contribute to the reaction along with the sample should be avoided.

Mention should be made also of the special requirements for the sample holders to be used with condensable gases. For example in the study of water condensation on cold 
surfaces it is important that the sample be the coldest spot in the high pressure chamber, otherwise simple thermodynamics considerations indicate that only a few layers of water or ice will form on the sample while the bulk of the water will condense on the coldest spot.

\subsubsection{X-ray windows}

The x-rays enter the high pressure chamber through a window covered by a membrane approximately 100 or $50 \mathrm{~nm}$ thick that prevents gases from entering into the $\mathrm{x}$ ray source, which typically operates under ultra-high vacuum. The membranes are made of $\mathrm{Si}_{3} \mathrm{~N}_{4}, \mathrm{Al}$, or $\mathrm{Si}$, with $\mathrm{Si}_{3} \mathrm{~N}_{4}$ being currently the most commonly used. The window is mounted at the end of a tube mounted on bellows for easy alignment with the $\mathrm{x}$-ray bay from the ring. It must be located as close as possible to the sample to minimize the distance traveled by the x-rays inside the reaction chamber. This is important particularly for XAS experiments at high pressure because the absorption edge of molecules in the gas and adsorbed phases overlap strongly. Currently the distance between x-ray window and sample is approximately $1 \mathrm{~cm}$, in a geometry like that shown in figure 5a.

The non-resonant attenuation of the x-ray beam intensity through the membrane needs to be as low as possible. The transmission through a $100 \mathrm{~nm}$ thick Al membrane for example varies between 70 and $95 \%$ for photon energies between 400 and $1000 \mathrm{eV}$ (see graph in figure $5 b$ ). Heat dissipation due to the absorbed radiation can be a problem in intense sources such as undulators in the Synchrotron. The heat generated by the highly focused x-ray beams can cause failure of the membrane, including melting in the case of Al, creation of pinholes and leakages, particularly near its edges where it meets the support plate.

\section{X-ray electron spectroscopies}

\subsection{XPS and XAS}


Two types of electron spectroscopies are most frequently performed in studies of surfaces in gas environments: (XPS) and x-ray absorption spectroscopy (XAS). In XPS $\mathrm{x}$-ray photons from a laboratory source (e.g, from $\mathrm{Mg}, \mathrm{Al}$ anodes) or from a Synchrotron storage ring, impinge on the sample and excite core electrons to high energy states above the vacuum level. The core hole, with energy $E_{1}$, subsequently decays either through an Auger process or through photon emission (fluorescence). In the Auger process an electron is emitted with kinetic energy $E_{A}$ while two holes in higher energy levels $\left(E_{2}, E_{3}\right)$ are left behind in the parent atom. The relation $\mathrm{E}_{\mathrm{A}}=\mathrm{E}_{1}-\mathrm{E}_{2}-\mathrm{E}_{3}-\Delta \mathrm{E}-\phi$, where the $\mathrm{E}_{\mathrm{i}}(\mathrm{i}=$ $1,2,3)$ are the core level binding energies, and $\phi$ the work function of the sample. A correction $\Delta \mathrm{E}$ of a few $\mathrm{eV}$, is added to account for changes in the binding energies due to the presence of the other holes. In XPS the electron from the excited core level is emitted with a kinetic energy $E_{k i n}=h v-E_{1}-\phi$, where $h v$ is the incident $\mathrm{x}$-ray photon energy, $E_{1}$ the core level binding energy.

An advantage of Synchrotron radiation sources is the very high photon flux they provide, several orders of magnitude higher that in conventional sources. Due to the small cross-section of the electron bunches circulating in the ring, synchrotrons are very bright sources, which makes high resolution imaging possible. For example, by using focusing zone plates, spot sizes of tens of nanometers have been achieved ${ }^{[18]}$. The technique known as scanning transmission x-ray microscopy or STXM, is currently at the forefront of spectroscopic imaging technologies, with applications in materials, environmental and chemical sciences.

When using home sources the energy of the x-rays is fixed, for example at $1430 \mathrm{eV}$ for $\mathrm{Mg}$ anodes and at 1250 for $\mathrm{Al}$ anodes, while with synchrotrons it can be varied in a continuous way. This very important feature opens the possibility to perform threshold excitation spectroscopies, where a core level is resonantly excited when the photon energy matches the difference between the energy of that level and that of the first unoccupied state. By scanning the photon energy one can explore the electronic structure of unoccupied levels, which is the basis of XAS, also called NEXAFS (for near edge xray absorption fine structure) or XANES (x-ray absorption near edge structure). Several books and reviews have been published ${ }^{[19]}$ on this subject. We will illustrate the use of 
XPS and XAS with several examples in this review. At photon energies above the ionization threshold, variations in absorption cross-section with energy give rise to characteristic oscillations in the spectrum. These are due to interference phenomena between outgoing electron trajectories scattering from the neighboring atoms. The oscillations contain information on the relative distances and number of the scattering centers near the parent atom. They are used in the technique called EXAFS (extended Xray absorption fine structure), for structural determination. This interesting topic however will not be covered here.

The absorption of X-rays, the basis of NEXAS and EXAFS, can be measured in several ways. On thin samples for example, one can measure directly the amount of transmitted radiation. On thick samples this is not possible and one must then measure some signal originating from the decay of the core holes. Fluorescence, the emission of $\mathrm{x}-$ rays, is a widely used technique. It has the advantage that the photons can travel through gases with little absorption and thus be easily detected by photodiodes. The disadvantage is that the information they contain is not very surface sensitive, unless the species being detected are only present at the surface. Also, because of the long traveling length inside the sample, differences in the absorption cross-sections with energy tend to attenuate, a phenomenon known as self-absorption. A more surface sensitive detection scheme is the so-called electron yield method, where electrons emitted by the sample are collected instead of fluorescence photons. These electrons, also called secondary electrons, are created through a cascade of multiple collisions where energy loss processes by primary photoelectrons and by Auger electrons create and redistribute electrons into an energy spectrum extending from the Fermi level to the energy of the primary photoelectrons. The emission can de directly measured with an appropriate detector outside the sample, or indirectly by measuring the sample to ground current.

Because electron attenuation in solids is hundreds of times stronger than that of photons, electron-yield spectra are much more surface sensitive. A more selective electron emission method is possible by using spectrometers to detect electrons emitted within a particular energy range, for example near the minimum of the mean free path curve, i.e., a few hundred $\mathrm{eV}$, or to correspond to emission from a particular element. For 
example Auger peaks of a particular species can be used for that purpose. Auger yield $\mathrm{XAS}$ as it is known, provides the highest surface and species sensitivity measurement.

Much like XPS, electron yield NEXAS in gas environments is possible by the techniques described in the previous paragraphs, i.e., with narrow apertures located at distances from the sample comparable with the mean free path of the electrons in the gas, and differential pumping schemes to separate electrons and gas molecules.

\subsection{Calibration and background signal issues}

As in every spectroscopy, quantification of the spectra is a very important task that requires careful experiments. For example to compare the intensities of XPS peaks from various elements the cross section for x-ray absorption needs to be known as a function

of energy. Fortunately reference data exist in the public domain ${ }^{[20]}$ that can be used for that purpose. Absolute coverage of species on the surface often requires the use of a reference material. For example in the case of water on $\mathrm{TiO}_{2}$ to be discussed below, the absolute amount of water was found by comparison of the water $\mathrm{O} 1 \mathrm{~s}$ peak intensity to that in the known (2x2) oxygen structure on $\mathrm{Pd}(111)$, measured in the same experimental conditions. A host of background subtraction and normalization procedures exist and used routinely in standard photoelectron spectroscopy.

Calibration of binding energies is also a delicate task, which can be performed by comparison with standards. For the spectra of the gas phase species located between sample and aperture, one has to take into account that changes in the work function of both sample and aperture material can shift the spectra. Since the geometry of the x-ray beam relative to the sample is grazing incidence and because the beam is only a few tens of micrometers in diameter, only the molecules located excited within that distance from the sample are excited.

An important aspect that is unique to photoelectron spectroscopy in gas environments is the absorption of x-rays by the gas phase. For example, in experiments where the gas pressure is varied, attenuation of the detected photoelectron intensity varies as well and needs to be determined and corrected. For that reason, often it is preferable to 
vary the chemical potential of the gas phase by varying the temperature while keeping the pressure constant. In this manner the correction for gas attenuation is the same for all data points.

Absorption of the x-rays by the gas phase, while less severe than that of electrons, can be a problem in XAS, particularly when the beam energy equals the white line threshold of the gas, which usually is very close to that of the adsorbed species. This problem is particularly important at high pressures, around 1 mbar and higher, which in the case of water is unfortunately one of the most important. Tedious background calibrations are then necessary, including obtaining separate spectra for the pure gas phase.

\subsection{Charging and beam damage effects}

A problem encountered in all electron spectroscopies in high vacuum environments is the charging of insulating samples. This problem is even more important when using synchrotron sources due to their higher beam intensity. A traditional way around this problem is to neutralize the sample by means of electron flood guns that send low energy electrons to the surface. Under pressures of even a few tenths of Pascal however, the small amount of ionized gas created by the incident $x$-rays is quite effective in discharging the samples, effectively playing a role similar to the flood gun. An example is provided in figure 6, corresponding to $\mathrm{KCl}$ under a water vapor pressure of $0.7 \mathrm{mbar}$. Under vacuum the same spectrum was strongly shifted in energy due to charging.

For very strong insulators, including alumina and mica, although large charging shifts are suppressed by the residual ions in the gas, the charge neutralization is not perfectly homogeneous and small patches or inhomogeneities in the sample charge distribution produce a broadening of the peaks that can be detrimental for high resolution spectroscopy.

A particularly relevant class of catalysts namely that of zeolites suffers in its analytical description badly from charging and differential charging. The surface 
composition and distribution of active sites being substitutions into the silicate framework structure were studied with great effort but only with limited success using conventional and synchrotron photoemission. These studies ${ }^{[21-24]}$ give a good account for the potentials and limitations of the surface analysis of non-conducting solids.

Another important problem that should be mentioned is the damage produced by the $\mathrm{X}$-ray beam. This damage is manifested in the preferential decomposition and ejection of electronegative species (the halide ions in salts for example), and the rupture of molecular bonds leading to decomposition of the surface species. These effects are contributed both directly by the x-ray photons and by secondary electrons from the substrate. Secondary electrons are the major culprits in beam damage effects. There is no easy solution to these problems. Minimizing exposure is of course a way to alleviate the problem. Another strategy is the continuous displacement of the sample so that new and different spots of the surface are hit by the beam for the shortest possible time. We found out that a combination of these strategies is quite effective in making the beam damage effects negligible.

A special case is that of liquid samples in equilibrium with their vapor. The rapid renewal of he surface layers by evaporation and condensation helps substantially in maintaining a fresh and unaltered surface.

\subsection{Gas phase spectra}

A unique advantage of electron spectroscopy under pressure in the millibar regime is that the gas phase molecules located in the path of the x-ray beam between the sample and the aperture can be detected by their XPS peaks. Fortunately the binding energies of gas phase species and that of species adsorbed at the surface are usually separated by several $\mathrm{eV}$, as we will see in the examples below. In the geometry of our instrument, where the x-ray beam has a cross section of 5 x $50 \mu \mathrm{m}$ impinging at a shallow angle to the surface of about 20 degrees, a gas sheet of thickness similar to the beam dimensions next to the surface is analyzed. This makes possible to determine the presence of gas phase reactant and product species and to follow their changes in concentration as a function of reaction parameters, providing a convenient and complementary way to mass- 
spectrometry for the analysis of the gas species. Due to the gas flow conditions and the geometry of the sample and aperture, calibration of the XPS peaks is necessary.

In the next sections we present examples of applications of APPES to problems of environmental science, where water is the most important element and to catalysis.

\section{Applications to environmental science}

Environmental science is one of the fields that is benefiting enormously from the development of APPES, because to accomplish its objectives it requires a thorough understanding of the structure and properties of surfaces, solids and liquids, in equilibrium with the environment at near atmospheric conditions. APPES opens the way for electron spectroscopy studies of liquid surfaces because, except for a few materials that have vapor pressures at the melting point in the $10^{-7} \mathrm{~Pa}$ range (e.g. In, $\mathrm{Pb}, \mathrm{Ga}$ ), the vapor pressure of most liquids is high and outside the range of applicability of electron based techniques. In the following subsections we show a few examples of applications to studies of water surfaces and the growth of thin water films.

\subsection{The melting of ice}

At the triple point of water near $0^{\circ} \mathrm{C}$, the pressure is 6.12 mbar. Since at present the instrument is capable of operating at approximately 10 mbar, saturation humidity can be reached by cooling to near $0^{\circ} \mathrm{C}$. Using this technique Bluhm et al. ${ }^{[25]}$ investigated the changes in the structure of the water surface through the melting transition, when the temperature varied from $-40^{\circ}$ to $+2^{\circ} \mathrm{C}$. Auger yield XAS at the O1s edge provided information on the LUMO structure of water near the surface, while the composition was monitored by XPS. It was indeed shown theoretically by Petersen et al. and experimentally by Nilsson et al. that the XAS spectrum depends strongly on the $\mathrm{H}$ bonding structure of water ${ }^{[26]}$. Qualitatively the argument is as follows. The threshold transition $1 \mathrm{~s} \rightarrow 4 \mathrm{a}_{1}$ in the isolated molecule involves $\mathrm{s}$ and $\mathrm{p}$ orbitals that make the transition dipole allowed. When water forms ice it becomes tetrahedrally coordinated with 4 other molecules through H-bonds. This modifies the hybridization of the LUMO orbitals such that they become more s-like in character. This translates in a decreasing of 
the absorption probability. The transition from ice to liquid is marked by the progressive increase in the number of broken H-bonds. Therefore it is the LUMO orbitals of molecules with dangling H-bonds (non-donor type molecules), that contribute the most to this transition. This allowed the authors to study the changes in H-bonding through the melting transition. The experiments were carried out on millimeter-thick ice films grown on a cold $\mathrm{Cu}$ substrate. The substrate was heated from liquid nitrogen temperatures to the melting point, and slightly above. Supercooled water was formed at $-2^{\circ} \mathrm{C}$ by slow cooling of the liquid film. The evolution of the XAS is shown in figure 7. The peak at $535 \mathrm{eV}$, corresponding to transition 1s $\rightarrow$ lowest LUMO state, increases appreciably in intensity from the ice to the liquid. The peak is already present in ice at low temperatures due to the surface molecules, which necessarily have dangling H-bonds. The gradual increase in intensity of this peak indicates that pre-melting is occurring. Pre-melting is characterized by an increase in the number of broken H-bonds in a thin film, sometimes referred to as being "liquid-like". Using the intensity of this peak (evaluated by a Gaussian fitting) and the attenuation length of $500 \mathrm{eV}$ electrons $(\sim 2.3 \mathrm{~nm})$ the thickness of the liquid-like film was measured, as shown in the graph of figure 8 . As can be seen, pre-melting starts to be visible around $-20^{\circ} \mathrm{C}$ and the thickness of the liquid-like layer increases rapidly above that, reaching $1.5 \mathrm{~nm}$ around $-5^{\circ} \mathrm{C}$ and quickly diverging as the temperature approaches the triple point value.

An interesting finding of this work was that the extent of pre-melting is influenced by the presence of surface impurities. Using XPS the authors could confirm that carbonaceous species are present on the surface due to submonolayer-level contamination. Some of these contain oxygen, like alcohols or other oxygenated compounds. The O-peak of these contaminant species contributes to the absorption peak at $532 \mathrm{eV}$ in figure 7. The finding is of importance in environmental sciences because organic contamination is always present and can influence the structure of the surface.

\subsection{The surface of aqueous ionic solutions}

APPES was recently used to investigate the surface composition of aqueous salt solutions, with the aim of determining the possible segregation of ions to the surface, an important problem in atmospheric sciences, where it has been predicted that reactions of 
sea-salt aerosols with gas-phase oxidants such as $\mathrm{OH}$ and ozone provide a mechanism for the production of substantial amounts of gas-phase halogen compounds in the troposphere. Experiments suggest ${ }^{[27,28]}$ and molecular dynamic simulations ${ }^{[29]}$ predict that large, highly polarizable anions $\left(\mathrm{Br}^{-}, \mathrm{I}^{-}\right)$segregate to the surface of salt solutions. An example of these simulations for the case of $\mathrm{NaBr}$ is shown in figure 9. It is predicted that the enhancement of anions at the surface of the aerosols leads to $\mathrm{Cl}_{2}$ formation and ozone depletion in the high regions of the atmosphere through sunlight activated chemistry ${ }^{[27}$, ${ }^{30]}$. Although $\mathrm{Cl}^{-}$is the main anion in aerosols of marine origin, there is also a considerable amount of $\mathrm{Br}^{-}$. In solid $\mathrm{NaCl}_{\mathrm{x}} \mathrm{Br}_{1-\mathrm{x}}$ alloys it has been shown that $\mathrm{Br}$ is highly concentrated at the surface after exposure to water vapor that causes dissolution of the surface layers ${ }^{[31]}$.

To ascertain this important prediction Ghosal et al. used APPES ${ }^{[32]}$ to study the dissolution of $\mathrm{KBr}$ and $\mathrm{KI}$ crystals. To access the $75 \%$ relative humidity value necessary to dissolve the crystals (the deliquescence point) within the limits of the apparatus $(<10$ mbar), the crystals were cooled to $-10^{\circ} \mathrm{C}$, where deliquescence was reached at 2.7 mbar. The energy of the x-rays was chosen so that the photoelectrons had the same kinetic energy of $160 \mathrm{eV}$ for both ions, ensuring that the sampling depth is the same for all elements and that surface sensitivity is maximal. Starting with the cleaved crystal in vacuum, the ratio of halide to alkali atom concentration was measured using the areas of the corresponding XPS peaks. The ratio remained constant and equal to 1 until the deliquescence point was reached (figure 10), where an abrupt increase to a value near 2 for $\mathrm{KCl}$ and 4.5 for $\mathrm{KI}$ was observed. A macroscopic drop was formed by the dissolution of the crystals, visible through the viewport as shown by the picture in figure 10. The higher segregation of I as compared with $\mathrm{Br}$ is in line with the predictions that large, more polarizable ions, segregate preferentially.

Another important result obtained by the authors is the variation of the ratio as a function of the kinetic energy of the photoelectrons. This is possible thanks to the tunability of the x-ray energy in synchrotron radiation. As the kinetic energy of the detected photoelectrons increases they can escape from deeper layers under the surface. In that manner a depth profile of the ionic concentration ratio was obtained. This is shown in figure 11 where the anion/cation ratio is plotted versus integrated electron 
escape depth (obtained from published mean free path tables) at the corresponding photoelectron energies. The plot also shows that segregation of the halide is restricted to the first three to five molecular layers with the ratio of concentration recovering the bulk value after $1.5 \mathrm{~nm}$.

\subsection{Water films on surfaces}

The growth and structure of water on the surface of materials is an important topic in environmental science because water films play a crucial role in corrosion, dissolution and rock weathering, catalysis and biological processes. APPES provides a unique tool to investigate many important questions that surprisingly are still poorly known today. For example, it is not yet known in general how much water is condensed on the surface of a material as a function of humidity. The structure of the water film is also poorly known including the chemical state, dissociative or molecular, of the first water layer. It is important to determine also the molecular orientation and the persistent length of any substrate-induced orientation into the bulk of the liquid. This is an important question because water structuring near biological interfaces can mediate the interaction between proteins, membranes, etc. In the next sub-sections two examples are shown to illustrate how the application of APPES can provide answers to some of these questions.

\subsubsection{Water on $\mathrm{TiO}_{2}(110)$}

The adsorption of water on $\mathrm{TiO}_{2}(110)$ under ambient conditions was studied by Ketteler et al. by monitoring the intensities of the XPS peaks in the $\mathrm{O}$ 1s region in the presence of water vapor ${ }^{[33]}$. As shown in figure 12 three peaks were resolved, originating from lattice oxygen at $530.4 \mathrm{eV}, \mathrm{OH}$ at $531.7 \mathrm{eV}$ and molecular water at $534 \mathrm{eV}$. At the higher pressure a peak at $536 \mathrm{eV}$ was also visible due to gas phase water. The bottom spectrum in the figure was obtained in the presence of $2.25 \mathrm{~Pa}^{\circ} \mathrm{H}_{2} \mathrm{O}$ at $298 \mathrm{~K}$ and the top one at $133 \mathrm{~Pa}$ and $270 \mathrm{~K}$. The amount of water on the surface was determined from the area of the XPS peaks, after calibration using the peaks of the well known $\mathrm{O}(2 \times 2)$ $\operatorname{Pd}(111)$ surface. 
The findings of the study can be summarized as follows. The titanium oxide surface has a small number or residual oxygen vacancy defects (about $0.2 \mathrm{ML}$ in that study). Water adsorbs first dissociatively in these vacancies giving rise to two chemisorbed hydroxyl groups, one from the water $\mathrm{OH}$ that fills the vacancy and the second created by reaction of $\mathrm{H}$ with bridge-bonded surface oxygen. This initial stage of the adsorption process occurs extremely fast, even for water pressures of $10^{-3} \mathrm{~Pa}$. Subsequent adsorption of water is molecular and occurs by $\mathrm{H}$-bonding to the $\mathrm{OH}$ groups that act as nucleation centers. The uptake of water is shown in figure 13(a) as function of relative humidity (RH). Since the same RH can be reached in isotherm or isobar experiments, a plot of coverage versus RH should be undistinguishable in both types of experiments if thermodynamic equilibrium has been achieved. As can be seen in figure 13 this is indeed the case. The film is about 3 monolayers thick between 20 and $50 \% \mathrm{RH}$, and about 8 monolayers around $95 \%$. It is only at the highest humidity values, within a few percent of saturation that the film thickness grows to macroscopic values.

One interesting finding of this study is that the $\mathrm{OH}$ groups are the nucleation sites for the growth of water because the molecules bind more strongly to these groups than to other water molecules. This was determined by direct measurement of the enthalpy of adsorption, which was found to increase from $70 \mathrm{~kJ} / \mathrm{mol}$ near $0.2 \mathrm{ML}$, to $45 \mathrm{~kJ} / \mathrm{mol}$, the value of the bulk liquid, when the coverage reached approximately $0.5 \mathrm{ML}$. The change in binding structure of water from the $\mathrm{OH}$ to groups to other water molecules is also manifested in the observed energy shift of the XPS peak of water to lower binding energies as the water coverage increases (see figure 12).

\subsubsection{Water on amorphous $\mathrm{SiO}_{2}$}

Silicon oxide is one of the most important materials in nature as part of many minerals, and in electronic technology, forming capping layers on $\mathrm{Si}$ wafers and in MEMS devices. It is therefore important to determine the structure and growth of water on this substrate. This was done recently by Verdaguer et al. ${ }^{[34]}$ on Si-wafers prepared using the RCA standard cleaning method. After cleaning, a new oxide layer was grown by exposing the surface to $\mathrm{UV} / \mathrm{O}_{3}$, which produced a highly hydrophilic surface, as 
shown by the complete spreading of a water droplet. Like in the $\mathrm{TiO}_{2}$ example, this wafer was analyzed by XPS while exposed to water vapor to pressures of up to 2 mbar, as shown in figure 14. The observation of the reduced Si2p peak indicates that the oxide film thickness is only a few monolayers. Oxygen from molecular water produced a peak located between that of the lattice and gas phase species. Its intensity was used to determine the thickness of the water film. At $100 \% \mathrm{RH}$ macroscopic condensation occurred and a visible film formed at the surface. As in the case of $\mathrm{TiO}_{2}$, the results from both isobar and isotherm experiments were the same, as expected from thermodynamic equilibrium.

In a separate experiment the change in surface potential of the sample was measured by AFM in the Kelvin probe mode to determine any preferential orientation of the water dipoles during growth. The results, plotted in the same graph as the uptake curve from the XPS experiments are shown in figure 15. The findings of these combined experiments can be summarized as follows: a rapid uptake takes place between 0 and $20 \% \mathrm{RH}$ leading to the formation of approximately 2 monolayers of water. The water molecule, probably bound to surface $\mathrm{OH}$ groups, is randomly oriented as shown by the invariance of the surface potential of the sample. Between 20 and 70-80\% RH, a slower increase in the rate of adsorption leads to the formation of 4-5 monolayers of water. Iit is in this range that the surface potential increases to its full and final value of $+0.4 \mathrm{~V}$. Above $80 \% \mathrm{RH}$ the amount of water increases rapidly to macroscopic amounts at $100 \% \mathrm{RH}$, while the contact potential remains constant. The increase in potential is in line with the fact that in bulk water the surface molecules have dangling H-bonds pointing to the vacuum. The authors concluded that the water layer on the $\mathrm{SiO}_{2}$ film reaches a bulk-like structure after 4-5 layers at room temperature. XAS experiments revealed that the structure of the water film was ice-like below $0^{\circ} \mathrm{C}$ and liquid-like above it, supporting the finding that the orientation effects of the first water layer, bound to the $\mathrm{OH}$ groups, do not propagate beyond the second layer.

\section{Applications to catalysis}


Catalysis science is greatly benefited by application of APPES because the technique can provide a direct insight into the chemical state of the catalyst during the reaction. For example a fundamental question is the oxidation state of the catalyst in oxidationreduction reactions, which can change rather rapidly depending on the gas mixture. APPES makes possible to construct phase diagrams for a wide range of pressure and temperatures, greatly expanding the range of pressures and temperatures where thermodynamic equilibrium can be achieved. This allows for a direct comparison with theory, where recent advances in computation and methods make it possible to calculate equilibrium phase diagrams from first principles ${ }^{[35,36]}$.

APPES provides direct information on surface composition, including reactants, products, contaminants and spectator species that might only be present under a steady state of exchange with the gas phase. In addition, the depth profiling capability inherent in the variable energy $x$-rays in synchrotron sources can be used to great advantage to explore migration of species into the subsurface region, which can affect the reactivity.

Finally, as already mentioned, APPES either in the XPS or XAS modes can detect also the gas phase species near the surface revealing in one single experiment the reactant and products in the vicinity of the surface, which can be used for example to monitor reaction rates. The following subsections illustrate these capabilities with examples from the author's laboratories.

\subsection{Oxidation of Pd(111)}

A recent in situ surface X-ray diffraction (SXRD) study has identified different crystalline phases of oxidized $\operatorname{Pd}(100)$ over an extended ${ }^{[37]}$ pressure and temperature range. For that reason new photoemission measurements ${ }^{[38]}$ were carried out on $\operatorname{Pd}(111)$ by Ketteler et al.under oxygen pressures up to $1 \mathrm{mbar}$. This spectroscopic study revealed for the first time the various steps leading to the complete oxidation of this transition metal.

Various phases of the O-Pd system were identified in addition the $(2 \times 2)$ chemisorbed phase, by their well defined XPS peaks in the O1s and Pd3d and Pd3p regions, as shown in figure 16. For $\mathrm{p} \geq 10^{-4} \mathrm{~Pa}$ and for $\mathrm{T}<750-900 \mathrm{~K}$, a two-dimensional monolayer thick 
surface oxide was observed. This phase has been found previously ${ }^{[39]}$ and is characterized by a LEED pattern corresponding to a $\sqrt{6} x \sqrt{ } 6$ superstructure. In XPS it is characterized by

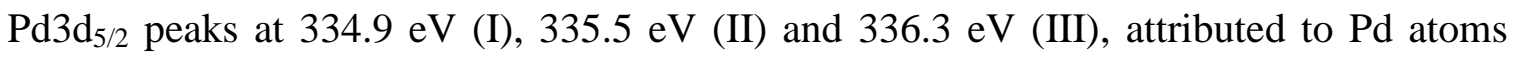
with two (II) and four (III) O neighbors ${ }^{[37]}$, and bulk Pd (I) ${ }^{[40]}$. The O1s region shows two peaks at 529.0 and $529.7 \mathrm{eV}$ with an intensity ratio of $\sim 1$, which have been assigned to two different oxygen species (threefold and fourfold coordinated $\mathrm{O}$ atoms). The stoichiometry of this surface oxide phase can be written as $\mathrm{PdO}_{0.3}$.

Between $10^{-2} \mathrm{~Pa}$ and $40 \mathrm{~Pa}$ and $\mathrm{T}<650-850 \mathrm{~K}$ the surface oxide transforms into a new phase characterized by XPS peaks at identical binding energy as in the surface oxide but with a $\mathrm{Pd} 3 \mathrm{p}: \mathrm{O} 1 \mathrm{~s}$ peak ratio corresponding to the stoichiometry $\mathrm{PdO}_{0.6 \pm 0.1}$ over the probed sample thickness. The ratio between the $\mathrm{Pd} 3 \mathrm{~d}_{5 / 2}$ peak areas at 336.3 and $335.5 \mathrm{eV}$ has increased from 1:4 in the surface oxide to values between $1: 2$ and $1: 1$, indicating that the fraction of highly coordinated $\mathrm{Pd}$ atoms (four $\mathrm{O}$ neighbors) has increased. Peak positions remained also unchanged in the $\mathrm{O} 1 \mathrm{~s}$ region, although the $\mathrm{O}$ intensity increased. These observations indicate that the chemical state of the $\mathrm{Pd}$ and $\mathrm{O}$ atoms is similar to that of the surface oxide phase. It was assigned to a two layer thick oxide and referred to as "subsurface oxide". Interestingly the subsurface oxide is formed always upon cooling in $\mathrm{O}_{2}$ only. When heating to reduce the oxide, the $\mathrm{PdO}$ phase transform directly into the surface oxide phase, indicating that the subsurface oxide is metastable. When the oxygen pressure increased to the mbar range $\mathrm{PdO}$ was formed. The $\mathrm{Pd} 3 \mathrm{~d}_{5 / 2}$ region shows one single peak, which is shifted by $\sim 1.6 \mathrm{eV}$ compared to bulk metallic Pd.

The experimental observations at many pressures and temperatures were used to construct the phase diagram shown in figure 17(a), where each point represents an independent experiment where no change was observed in the spectra over several minutes. Four different regions can be observed in the diagram. The top left corresponds to the high-vacuum, high temperature, chemisorbed $2 \times 2$ phase. Below this, the green area represents $p, T$ conditions where the monolayer surface oxide phase is stable. The $p, T$ region where the new "subsurface oxide" was observed is marked blue, and the bulk PdO in dark. Finally, the dark region corresponds to the PdO bulk oxide when the pressure is reduced from 1 mbar at fixed temperature. 
The solid line indicates the phase boundary between $\mathrm{Pd}$ and $\mathrm{PdO}$ as calculated by tabulated values of the enthalpies and heat capacities ${ }^{[41]}$. As can be seen the boundary agrees well with that observed between $\mathrm{PdO}$ and the surface oxide at the higher temperature. However the surface oxide phases are not predicted by classical standard thermodynamics. The surface phase diagram calculated by atomistic thermodynamics ${ }^{[36]}$ is shown in the bottom phase diagram (b). It is clear that kinetic limitations are present that prevent reaching thermodynamic equilibrium at the lower temperatures.

One of the most interesting results of this study is the observation of the existence of the metastable subsurface oxide, kinetically stabilized by the large activation energy separating the clean metal and surface oxide phases from bulk PdO. Its origin is likely to be the large crystallographic restructuring needed to go from the metal to the bulk oxide. As a result the less stable surface oxide structure, which is in epitaxial relationship with the metal substrate can grow into a two (and perhaps more) layers thick film before finally transforming to PdO. Support for this idea was obtained from kinetic measurements, from which the authors derived the activation energies of the various transformations, shown in figure 18. The activation energy for the transformation of the surface oxide to the bulk oxide was found to be more than twice that for the formation of the surface oxide. The reduction from the bulk oxide directly to the surface oxide bypassing the subsurface oxide however, has the same activation energy.

The growth of such metastable epitaxial oxides might be a rather general phenomenon in the oxidation of metals. As the surface free energy of oxides is lower than that of metals in most cases, oxides are expected to wet the metal surface, assuming that the interface free energy is negligible. In most cases however, the interface energy is too large due to the lattice mismatch between the oxide layer and the metal.

\subsection{Redox Chemistry of Cu: Oxidation of $\mathrm{Cu}_{2} \mathrm{O}$ to $\mathrm{CuO}$}

The oxidation of copper has been studied extensively on single crystals ${ }^{[42-44]}$ and alloy systems ${ }^{[45]}$. Surface oxide films have been found to form in parallel with extensive dissolution and sub-surface oxidation processes. The difficulty of distinguishing the $\mathrm{Cu}$ valence between 0 and +1 , coupled with the semiconducting nature and defect structure 
of its oxides do not allow for suitable referencing. It is thus desirable to determine the spectral properties of stoichiometric oxides with high resolution spectroscopy. This is possible using APPES because the in-situ oxygen gas environment prevents artificial reduction of the beam-sensitive $\mathrm{CuO}$ and prevents formation of hydroxyl groups from background water or hydrogen.

The experiment was performed both on a $\mathrm{Cu}(111)$ crystal and on a polycrystalline foil. The sample was first heated to $573 \mathrm{~K}$ in 0.25 mbar of oxygen to produce $\mathrm{CuO}$ followed by reduction in hydrogen to produce $\mathrm{Cu}_{2} \mathrm{O}$. Annealing the $\mathrm{Cu}_{2} \mathrm{O}$ in $0.25 \mathrm{mbar}$ oxygen from $473 \mathrm{~K}$ back to $573 \mathrm{~K}$ produced pure $\mathrm{CuO}$. The O 1s XPS peaks obtained during this process are shown in Figure 20. The gas phase $\mathrm{O}_{2}$ peaks, visible on the left hand side illustrate the good resolution obtained and provide a reference for the binding energy scale. The oxide peaks show clear differences in binding energies in the two valence states, which can be explained by the fact that the $\mathrm{O}^{2-}$ anions interact covalently and differently with the open d-shell $\mathrm{Cu}$ ions in $\mathrm{CuO}$ and the formally closed d-shell $\mathrm{Cu}$ ions in $\mathrm{Cu}_{2} \mathrm{O}$. After 30 min of heat treatment the transformation is complete although a new peak, at around $532 \mathrm{eV}$, appears that indicates deterioration of the structure. In the absence of any sign of hydroxyl in the valence band spectra and considering the abundance of molecular oxygen in the gas phase this line is assigned to oxygen bonded to defective surface sites. The high binding energy is characteristic of electrophilic atomic oxygen ${ }^{[46,47]}$ and is not indicative of a molecular species, which on the other hand is very unlikely to be present at $573 \mathrm{~K}$ in the abundance necessary to produce the observed intensity.

\subsection{Reduction of a Cu/ZnO nanostructured system}

In the synthesis of high performance catalysts, thermal treatment steps (calcinations, reduction, activation) in various gas atmospheres are crucial operations for the performance and stability of the resulting nanostructured materials. Yet their analytical evaluation is still very poor giving rise to the notion of "black magic" in the preparation of good catalyst materials. Amongst the over 2000 reviews listed in the ISI database on 
catalyst synthesis and preparation there is not a single one describing in-situ methods for studying the preparation of materials.

The $\mathrm{Cu} / \mathrm{ZnO}$ catalyst for methanol chemistry ${ }^{[48,49]}$ is prepared from a precipitated carbonate precursor that is calcined to form an oxide mixture and finally reduced to $\mathrm{Cu}$ metal with $\mathrm{ZnO}$. The practical composition $\mathrm{Cu} 70 \mathrm{Zn} 30$ precludes the notion of a supported system and calls for a diluting, yet critical function, ${ }^{[50,51]}$ of the $\mathrm{ZnO}$. The $\mathrm{Cu}$ nanoparticles of about 4-7 nm diameter undergo dynamic restructuring as function of the chemical potential ${ }^{[52,53]}$ and do not significantly sinter due to the spacer function of the $\mathrm{ZnO}$. The issue of brass formation as origin of the "synergistic" interaction between $\mathrm{Cu}$ and $\mathrm{ZnO}$ has been discussed controversially ${ }^{[54]}$ without definitive experimental evidence under in-situ conditions.

A critical issue of this and many other synthesis of metal particles from oxide precursors is the possibility of residual oxygen content. It is usually possible by using XRD and EXAFS ${ }^{[50,55]}$ to determine the purity of a metal particle phase to about $1 \%$. Oxygen concentrations in the ppm range can, however, have significant effects on the electronic and geometric properties of metals as they induce strain. They can also change the local electronic structure of e.g. copper ${ }^{[50,56]}$ much below the threshold of "chemical oxidation" to a discernible oxide phase. Such a situation was studied recently in the case of $\mathrm{Ru}$ oxide catalysts ${ }^{[57]}$. Oxygen contents below the oxide formation limit ${ }^{[42]}$ would allow for tuning electronic properties of catalysts and would stabilize metastable surface structures ${ }^{[58,59]}$ of nanoparticles, as in the case of Pd discussed above ${ }^{[32]}$. Unintentional consequences of thermal treatments used to "control" the residual oxygen content of metal catalysts, may modify the oxygen content by thermal decomposition of oxide components of the catalysts (support) and/or by incorporation of tiny amounts of oxygen from impure gas phases present during calcination. These effects would explain a good deal of the "black magic" in the preparation of metal nanoparticle catalysts. In a recent review ${ }^{[60]}$ addressing the synthesis of metal nanostructures from oxide precursors the important phenomena due to trace amounts of residual oxygen were omitted. The authors used single crystal samples that tend to exhibit a kinetic delay in their response to the chemical potential of the gas phase leading to sudden phase changes of highly metastable states. The resulting "simple" sequence of phase transformations suppresses 
the continuous responses of the solid to the chemical potential observed in nanostructures with their low kinetic barriers to structural changes ${ }^{[61]}$. The kinetic anisotropy observed when going from metal to oxide as compared from oxide to metal causes hysteretic effects in phase transitions of catalytic metals ${ }^{[62-64]}$ and allows for metastable systems to be in existence during catalysis after low-temperature preparation of the functional material. It may thus well be that a metal with residual oxygen content is present in nanoparticular form in a hydrogen-containing atmosphere.

$\mathrm{A} \mathrm{Cu} / \mathrm{ZnO} 70 / 30$ binary catalyst precursor was calcined as described in the literature [50,55] and subject to reduction in 0.25 mbar of hydrogen at temperatures up to $523 \mathrm{~K}$, representing the operation temperature of such catalysts in steam reforming and methanol synthesis. Both reactions are closely related to each other via the water gas shift reaction. The reduction of the technical catalyst occurs at a hydrogen pressure of 50 mbar and is completed according to thermal gravimetry at around $500 \mathrm{~K}$. As the pressure gap between technical and in-situ reduction is still substantial, yet 6 orders of magnitude reduced compared to that in surface science experiments, it will be the temperature of reduction that indicates the relevance of the in-situ experiment.

Figure 21 shows the evolution of the $\mathrm{Zn} 3 \mathrm{p}$ and $\mathrm{Cu} 3 \mathrm{p}$ XPS signals during reduction from $300 \mathrm{~K}$ to $453 \mathrm{~K}$. The sharpening of the p-doublet for $\mathrm{Zn}$ is due to a gradual transformation of $\mathrm{Zn}$ hydroxy-carbonates into $\mathrm{ZnO}$, as evidenced by comparing XPS and $\mathrm{L}_{2,3}$ XAS from reference compounds. In these example XAS is more chemical statespecific than XPS shifts, although the later can be quantified much more readily. XAS clearly showed that at the end of the heating step the $\mathrm{Cu}$ in the sample was converted to $\mathrm{Cu}_{2} \mathrm{O}$ and not into $\mathrm{Cu}$ metal, which would exhibit the exactly same XPS signature as that in Figure 21. This finding is in agreement with in-situ bulk EXAFAS measurements ${ }^{[65]}$ and reveals that the synthesis of the catalyst has to go through the intermediate $\mathrm{Cu}_{2} \mathrm{O}$. It is interesting to compare this finding to literature results, where formation of $\mathrm{Cu}_{2} \mathrm{O}$ was not recognized after the hydrogen reduction of $\mathrm{CuO}^{[55]}$. The controversy can be resolved by recognizing the important role of kinetic effects. Depending on the details of the nanostructure of the oxide and the severity of the reduction condition, intermediate species may or may not accumulate and form separate phases. The detection of such intermediate forms is of importance in understanding the nanostructure of the final $\mathrm{Cu}$ 
metal particles because the different lattice parameters of the two oxides co-existing in the reduced material can produce strain at their interface, which is the driving force for the production of other defects and irregularities in the final metal structure.

The agreement between bulk and surface analysis at various stages of the reduction process shows that the hydrogen pressure results in sufficient chemical potential to produce the same phase at the surface and within the initial oxide particles. Thus, the pressure gap between real and in-situ analytical conditions should have no qualitative influence on the results.

At higher temperatures a significant change of the oxygen 1s peak was observed, while only small changes occurred in the metal spectra. Figure 22 shows the XPS O1s data together with the in-situ mass spectrometric traces for the reduction products, water and $\mathrm{CO}_{2}$, produced by the incomplete calcination of the carbonates. The lines in the figure, marking the positions of the oxygen binding energies for the two copper oxides, reveal that at no time in the process pure copper oxides are present. This explains the necessity to go at length into the preparation of precursors if a metal supported on an oxide is the desired product. The intuitive assumption that a mixture of precursor oxides would bring about the desired $\mathrm{Cu}$ on $\mathrm{ZnO}$ system is incorrect as the reduction kinetics of the system must be set such that only $\mathrm{Cu}$ and not the also easily reducible $\mathrm{ZnO}$ is affected by the hydrogen chemical potential. The intimate nanoscopic mixture of the precursors allows for the preferential reduction of $\mathrm{Cu}$, as expected from their chemical potential $(\mathrm{Cu}$ is nobler than $\mathrm{Zn}$ ), while $\mathrm{ZnO}$ exerts a diluting function during the formation of the nanoparticles. This selection is achieved by producing defective $\mathrm{Cu}$ oxide precursors as evidenced in the present in-situ experiments. It is noted that this information cannot be gained from X-ray diffraction data ${ }^{[51]}$ revealing only the presence of defective $\mathrm{CuO}$ and of some species giving rise to background distortions.

The result is a 3-dimensional interlinked network of $\mathrm{Cu}$ nanoparticles ${ }^{[55,66]}$ connected by $\mathrm{ZnO}$ binder. The process of nanostructuring begins at the end of the heating period, at $523 \mathrm{~K}$, when the new $\mathrm{O} 1 \mathrm{~s}$ peak of $\mathrm{ZnO}$ appears between those of the mixed oxides and carbonates, as shown in Figure 22. From the mass-spectral traces it can be seen that the initiation of the copper formation process is coupled to the maximum rate of carbon dioxide loss indicating the crystallization of $\mathrm{ZnO}$ that holds the $\mathrm{Cu}$ nanoparticles 
in place. For the topochemistry of the copper formation it is important to know that at this moment $\mathrm{Cu}$ is present entirely as $\mathrm{Cu}_{2} \mathrm{O}$, as seen from the data in Figure 21, showing that this oxide it is more difficult to reduce than $\mathrm{CuO}$. This is key to understand that at the end of the holding time at $523 \mathrm{~K}$ the reaction may still be incomplete, despite the fact that pure metallic copper has been formed according to structural analysis ${ }^{[65]}$ and that the data in Figure 22 seem to indicate complete conversion.

At the end of the reduction process a catalytically active surface is obtained (see below) with an $\mathrm{O} 1 \mathrm{~s}$ spectrum showing $\mathrm{ZnO}$ at low binding energy and some residual basic or carbonate species at higher binding energies. A consequence of the nanostructural transformation is that sharply at the end of the reduction process the atomic ratio of $\mathrm{Cu} / \mathrm{Zn}$ changed drastically. Figure 23 reports the behavior deduced from the XPS data. At the end of the $\mathrm{CuO}$ reduction and after evolution of most of the $\mathrm{CO}_{2}$ (arrow) a rapid decrease of the $\mathrm{Cu} / \mathrm{Zn}$ ratio occurs. It is unclear from the line shapes of the species involved if the surface of the $\mathrm{Cu}$ gets covered with $\mathrm{ZnO}$ (likely as no changes in profiles occurred) or by brass. Zn metal formation is unlikely since due to the high dispersion, a spectrum of $\mathrm{Zn}$ metal should be discernible besides the prevailing $\mathrm{ZnO}$ spectrum. The delay between the end of reduction and the change in surface composition may be caused by insufficient energy transport to the surface to compensate the strong cooling caused by desorption of water. The alternative explanation of a dispersion change is not valid as the composition does not allow for a large modification of the $\mathrm{Cu}$ particle size (there is no sufficient "support") and the XRD/EXAFS data ${ }^{[55,65,66]}$ indicate no change in the particle size after calcination. The result of the reduction process is thus a system of $\mathrm{Cu}$ metal covered in part (about 50\%) with $\mathrm{ZnO}$.

The valence state of $\mathrm{Cu}$ at the surface is of great interest to understand its catalytic function. XPS is unsuitable to detect a possible mixture of $\mathrm{Cu}_{2} \mathrm{O}$ and $\mathrm{Cu}$, and the existence of $\mathrm{ZnO}$ in high dispersion precludes analysis of the oxygen $1 \mathrm{~s}$ data, even when a shift between oxygen in $\mathrm{Cu}$ and in $\mathrm{ZnO}$ is likely to exist. Figure 24 shows in-situ XAS data obtained at the beginning and end of the reduction process. Data from the reference experiments described in Figure 20 were used for comparison. At the beginning $\mathrm{Cu}$ is present as an open d-shell $\mathrm{CuO}$ compound resulting in large white-line intensity. The small contribution from d-states in the nominally close d-shell compound $\mathrm{Cu}_{2} \mathrm{O}$, caused 
by covalent interaction, gives rise to the shifted and much weaker white line seen for the reference compound. The final reduction product is chemically very close to metallic copper as all signatures of the reference spectrum are fully developed in the spectrum of the ca. $5 \mathrm{~nm}$ large catalyst particles. It is unlikely that some of the particles were simply not fully reduced under the conditions applied.

Taking into account the presence of sub-surface oxygen species, as detected in earlier studies ${ }^{[67]}$, together with the kinetic difficulty discussed above to reduce $\mathrm{Cu}_{2} \mathrm{O}$, the data indicate an "impure Cu metal" and the presence of chemically non-reactive subsurface oxygen that modifies the electronic structure of the $\mathrm{Cu}$ nanoparticles. Model experiments with strained $\mathrm{Cu}$ layers ${ }^{[56]}$ revealed the existence of such electronically modified $\mathrm{Cu}$ as a consequence of (mechanically) applied strain only in the presence of some oxygen. Exactly these conditions apply here, due to crystal lattice mismatch between $\mathrm{Cu}$ and $\mathrm{Cu}_{2} \mathrm{O}$. Unfortunately it cannot be resolved at this point whether the presence of $\mathrm{ZnO}$ favors the existence of the oxygen at the surface that creates or maintains the strained state of the $\mathrm{Cu}{ }^{[54,68]}$. It is clear that a "dirty" or metastable form of $\mathrm{Cu}$ nanoparticles is the result of such process. The old idea of paracrystals of oxide embedded in the metal ${ }^{[69][70]}$ and recently rejuvenated ${ }^{[60]}$, seems a less realistic description of the metastable state than the concept of a strained surface stabilized by sub-surface oxygen ${ }^{[44]}$. In studies of similar catalysts using a transfer reactor and XPS ${ }^{[71,}$ ${ }^{72]}$ it was also found that after reduction and after use, the majority of $\mathrm{Cu}$ was present as metal, with hints from a shifted modified Auger parameter that some "oxidic species" may be present.

The in-situ study is in line with a concept of active $\mathrm{Cu}$ as being composed of electronically modified $\mathrm{Cu}$ particles, prevented from sintering by $\mathrm{ZnO}$. The presence of about $25 \% \mathrm{Cu}$ at the surface at the end of the reduction process deduced from XPS (Figure 23), is in good agreement with determinations of the total $\mathrm{Cu}$ surface area by gas adsorption techniques, which report about $30 \%$ of the geometric surface as being $\mathrm{Cu}^{[73]}$. The agreement between results obtained with radically different methods supports the validity of either analysis, with the advantage that XPS is element-specific and requires no assumptions about the course of chemical reactions that were debated ${ }^{[74]}$ in the literature. 
A negative consequence of the granular structure of the samples is that the nondestructive depth profiling provided by synchrotron-based XPS, through varying the excitation energy, is not applicable. A flat photon energy-dependence of the $\mathrm{Cu} / \mathrm{Zn}$ ratio was found both in hydrogen and in methanol steam reforming conditions, even after ca. $100 \mathrm{~h}$ time on stream and under varying catalysis conditions. It can be concluded that the structural relation of $\mathrm{Cu}$ and $\mathrm{ZnO}$ remains stable after its formation under the conditions of the present experiments.

An apparent loss in resolution is observed in the final spectrum in Figure 22 at exactly the point where the $\mathrm{ZnO}$ spreads over the freshly formed copper. This is might indicate that the composition of $\mathrm{ZnO}$ is different in the spacer material and in the overlayer film. The very rich defect chemistry of $\mathrm{ZnO}^{[75]}$ allows for such subtle distinctions. The assumption of different defect states of $\mathrm{ZnO}$ filling the spaces between particles and on the $\mathrm{Cu}$ particles helps explain the apparent discrepancy of the present model with the observations by electron microscopy ${ }^{[76]}$ of a weak wetting interaction between $\mathrm{Cu}$ and $\mathrm{ZnO}$ without mentioning the possibility of its coexistence with a strongly wetting component of $\mathrm{ZnO}$. Here the in-situ XPS provides complementary insight of a thin and strongly wetting $\mathrm{ZnO}$ that would not be visible in the TEM.

\subsection{Catalytic reactions}

\subsubsection{Copper in $\mathrm{MeOH}$ oxidation}

Methanol is technically oxidized to formaldehyde either in a high temperature process at $973 \mathrm{~K}$ over metallic silver or in a low temperature process at $673 \mathrm{~K}$ over ironmolybdate oxide systems. Copper with its facile redox chemistry between oxide and metal, accessible at low and intermediate pressures ${ }^{[77]}$, is a suitable system for mechanistic studies of selectivity control. The fact that extensive kinetic oscillations in

methanol oxidation were reported is an indication ${ }^{[78]}$ that active $\mathrm{Cu}$ may exist both as an oxide and as a metal. In initial experiments it was found that clean copper without subsurface oxygen is inactive and that stoichiometric $\mathrm{CuO}$ (black) is also inactive, whereas red oxides $\left(\mathrm{Cu}_{2} \mathrm{O}\right)$ are active with maximum activity in transient regions where several 
phases co-existed due to kinetic instabilities ${ }^{[42,67]}$. This behavior was also found in $\mathrm{Ru}$ ${ }^{[79]}$, oxidizing $\mathrm{CO}$ at very high rates when the surface composition is ill-determined with respect to thermodynamic ${ }^{[80]}$ surface phases. It is thus of interest to know how the surface of a metallic catalyst responds with different surface phases according to the oxidizing or reducing nature of the gas environment, given by the partial pressures of $\mathrm{O}_{2}$ and $\mathrm{CH}_{3} \mathrm{OH}$. It can also be expected that an oscillatory change between oxide and metal, as observed at high pressures ${ }^{[78]}$ and in forced modes at low pressures ${ }^{[77]}$, may give rise to a spatially inhomogeneous distribution of oxidation states, which could not coexist in thermodynamic equilibrium. In such a situation the usual practice of performing the reaction in a reactor chamber and later transferring, after cooling and evacuation, into the UHV chamber for analysis will not show the correct surface state but rather the equilibrated state ${ }^{[42]}$ produced during evacuation. Ambient pressure in-situ analysis is thus crucial to find a valid and relevant answer that can serve as guideline for fundamental experimental and theoretical studies.

In our laboratories the catalytic oxidation of methanol was performed over $\mathrm{Cu}(111)$ and over polycrystalline $\mathrm{Cu}$ foil. The obtained results ${ }^{[81]}$ were fully consistent with earlier in-situ XAS studies ${ }^{[82-84]}$ that could not be quantified with respect to the abundance of species involved. The XPS results, in figure 25, show both surface and gas species, with the gas phase species appearing at higher binding energies due in part to the lack of relaxation contributions from the substrate electrons, which affect the surface species. In-situ product formation could be monitored both by XPS and by mass spectrometry. Up to four species of oxygen can co-exist on the reacting copper surface. One species forms patches of $\mathrm{Cu}_{2} \mathrm{O}$ and suppresses partial oxidation. The oxide can be reduced back to the metal at high chemical potentials of methanol, giving rise to combustion and $\mathrm{CO}_{2}$ formation. A second species, identified based on depth profiling, is a "surface oxide" that is not correlated to the catalytic activity. At high coverage this oxide suppresses the catalytic activity. A peak at $532 \mathrm{eV}$ was assigned to hydroxyl species strongly adsorbed to electrophilic $\mathrm{Cu}$ sites that arise from sub-surface oxygen and/or from edge/defect sites of the $\mathrm{Cu}_{2} \mathrm{O}$ patches. The other two species are surface atomic oxygen and bulk-dissolved oxygen. These two species were found on surfaces 
exhibiting a clear Fermi edge in the valance band spectra. Some experimental evidence is given in Figure 25.

The reaction on $\mathrm{Cu}(111)$ was carried out in methanol-rich gas (highly reactive). The reactivity data reveal a characteristic ignition-type activity curve. It is also seen that total oxidation of methanol under stoichiometric reduction of the $\mathrm{CuO}$ precedes partial oxidation confirming the inactivity of the oxygen-rich surface termination of the surface oxide or $\mathrm{Cu}_{2} \mathrm{O}$ for catalysis. The dominating peak of hydroxyl accumulating on the hot surface is a strong hint to the non-metallic nature of the surface, as pure $\mathrm{Cu}$ metal should not bind $\mathrm{OH}$ at such high reaction temperatures.

One of the most interesting findings of the study was the important role played by sub-surface oxygen. This can be demonstrated by the structure-function correlation graph shown in Figure 26. The integrated peaks for oxide and sub-surface oxygen correlate strongly with the production of formaldehyde in a positive mode for the sub-surface species and in a negative mode for the oxide. The positive correlation of the sub-surface oxide with formaldehyde production excludes a Mars-van-Krevelen ${ }^{[85]}$ mechanism, where this oxygen would be a reagent and thus consumed with higher production. It rather indicates that this oxygen acts as a proxy for the active site containing copper atoms and reactant oxygen species in such low steady state concentration that its signal cannot be resolved in the spectrum. This follows from the failure of any other spectral component in Figure 25 to correlate with the formaldehyde production. The negative correlation of the oxide component with $\mathrm{CO}_{2}$ production is an indication for its stoichiometric involvement, with oxide oxygen being consumed ${ }^{[85]}$ as a reagent and responsible for combustion. Through this function it is clear why the presence of oxide slows down the catalytic production ${ }^{[81]}$ of formaldehyde. It is reassuring to compare the structure-function plot derived from the data of Figure 25 to that derived from a much wider variation of parameters of the same reaction performed in a different instrument and at much better spectral resolution ${ }^{[81]}$. The results are strikingly similar and confirm the role of sub-surface oxygen in $\mathrm{Cu}$ as a co-catalyst for partial oxidation of methanol with an essential function. Sub-surface oxygen is thus not an unavoidable and irrelevant complication of high-pressure experiments. 
The assignment of the oxygen species with binding energies around $530 \mathrm{eV}$ as subsurface was unambiguously supported ${ }^{[81]}$ by non-destructive depth profiling. This assignment should be contrasted with the low pressure studies carried out in-situ ${ }^{[77]}$, which also summarize the extensive work of static experiments ${ }^{[86,87]}$ in the $\mathrm{Cu} / \mathrm{O} / \mathrm{MeOH}$ system. There is good agreement that at all pressures studied, copper oxide islands at metal surfaces are non-reactive and exhibit a binding energy of the oxygen species of $530.5 \mathrm{eV}$. In the energy range where the high-pressure XPS reveals the presence of subsurface oxygen, several other species were detected in the low pressure and low temperature experiments. There is agreement that a peak found at $529.6 \mathrm{eV}$ corresponds to adsorbed oxygen ${ }^{[77,88,89]}$. Whereas one study qualifies this oxygen as non-reactive ${ }^{[77]}$ another assigns it to high reactivity. Reactive oxygen should be found at $528.9 \mathrm{eV}$ according to the in-situ low pressure study ${ }^{[77]}$.

These apparently contradictory results could be reconciled in the following way. Reactivity towards adsorbents can be different for alcohols requiring nucleophilic oxygen and for $\mathrm{CO}$, ammonia or olefins that require electrophilic oxygen. In any case atomic oxygen must be present at the surface to be relevant as oxidant. The difference between nucleophilic (strongly bound on bare metal) and electrophilic (weakly bound on metal modified by strain or sub-surface oxygen) is determined by the composition of the adsorption site. At elevated pressure there will be always sub-surface oxygen that may be absent at low-pressures. The range of binding energies for surface and sub-surface oxygen species is so narrow $(528.5-530 \mathrm{eV})$ that, given the line width of the O1s peak of about $1.5 \mathrm{eV}$, discrimination of the various species is difficult. The depth profile of the sub-surface species has shown that under reaction conditions the oxygen concentration is highest at the interface ${ }^{[81]}$. In this location it can exert three functions:

As a site where methanol is adsorbed it may act as nucleophile and abstract protons leading to strongly bound hydroxyl groups. ${ }^{[82]}$

If surface atomic oxygen is adsorbed next to a sub-surface oxygen species this surface oxygen will be held only weakly to the surface due to the reduced electron density of the metal being affected by the sub-surface species. This will cause an electrophilic reactivity pattern leading to oxygen addition to methoxide and thus to total combustion. ${ }^{[46,47]}$. 
Oxygen segregating from the sub-surface to the surface can form ordered superstructures and a "surface oxide" ${ }^{[77]}$ that passivate the catalyst ${ }^{[87]}$ as the methanol molecules cannot bind between the oxygen species.

A summary of this situation is given in Figure 27 indicating that at low chemical potential as typical for surface science experiments only a fraction of these $\mathrm{Cu}-\mathrm{O}$ interactions are detectable. The conceptual simplicity of low oxygen chemical potential experiments, which made possible the discovery of the reaction mechanism via methoxy and formate ${ }^{[90]}$ on $\mathrm{Ag}$ and on $\mathrm{Cu}^{[88]}$, is counterbalanced by missing out of qualitative features ${ }^{[56]}$ comprising the material gap in this reaction.

The high-pressure experiment with its qualitatively different species as compared to the low potential experiment cannot exclude of the co-existence of the low- $\mu$ oxygen species with those present at high- $\mu$. The former may the precursors to the latter ones and thus exist only in low steady state abundance, or they may co-exist with sizeable coverage and contribute to the catalytic reaction that would have to deal with several species of electrophilic and nucleophilic oxygen. The structural feedback of lattice strain caused by sub-surface oxygen, interfering with the local electronic structure of the adsorption sites directly, and indirectly by creating steps and roughness and thus generating active sites ${ }^{[91]}$, couples the chemical potential of oxygen into the details of the electronic structure of all species. This makes it extremely difficult to determine the number of participating species from an analysis of the overall kinetic parameters or from performing low-pressure reaction studies. The feedback loop displayed in Figure 27 is the concrete answer to the "gaps" in this particular case. The debate about the nature of the exact species being reactive is resolved by accepting that under operating conditions not any single oxygen species with a sharply defined electronic structure is active but rather a distribution of species both in static electronic structure (width of XPS lines) and in residence time (abundance detectable within the time window of experimental data accumulation) will be found.

\subsubsection{Ruthenium: oxidation of $\mathrm{CO}$ and $\mathrm{MeOH}$}


As the spectral resolution of the copper-oxygen system seems not to be adequate enough to evaluate the issues posed with the scheme in Figure 27, it is useful to revert to another system exhibiting higher resolution of the spectral features. Such a system is provided by the oxidation reactions of $\mathrm{CO}$ and $\mathrm{MeOH}$ over $\mathrm{Ru}$, which has been studied extensively in the past as well as recently. The availability of extensive studies of both reactions is beneficial for the description of the nature of active oxygen.

From the nature of the substrates it is clear that $\mathrm{CO}$ oxidation probes the presence of electrophilic oxygen only whereas methanol oxidation also requires the presence of nucleophilic oxygen for formation of methoxy and formate. This discrimination of atomic oxygen with respect to the Lewis-acid base properties of the substrate is not always consistently applied in more physical inclined studies considering only "reactive" and "non-reactive" atomic oxygen.

The CO oxidation has been extensively reviewed from the experimental ${ }^{[92]}$ and theoretical ${ }^{[79,80]}$ viewpoints. It is highly consistent with the microkinetic model ${ }^{[93]}$ and its phase diagram is in agreement with the kinetic findings. It is now clear that both on single crystals as well as on supported nanostructured Ru the catalyst is a thin oxide film with a structure similar to $\mathrm{RuO}_{2}{ }^{[57,94]}$. This structure can have different terminations ${ }^{[95]}$ exposing in the present terminology electrophilic and, at high oxygen potential, also nucleophilic oxygen plus metal sites. There is also a rich sub-surface chemistry of bulk dissolved and in-surface embedded oxygen species whereby the latter being a strongly nucleophilic species is passivating the surface for CO oxidation ${ }^{[96-99]}$ as no electrophilic oxygen is present.

This system has been studied with high pressure XPS ${ }^{[100-102]}$ in parallel with imaging photoemission microscopy that provides information about the lateral distribution of species. It is indeed known from STM and PEEM studies that extended features ${ }^{[103]}$ and inhomogeneities are always present. The fact that the experiments could be carried out under pressures where steady state could be reached without the need to transfer to a separate analysis chamber makes possible an accurate analysis of the different states, irrespective of their stable or metastable nature. This is very important because after cooling and evacuation there is always the danger of phase segregation. 
An important result from APPES is the determination of the binding energies of many species with respect to the Fermi edge with a resolution of $0.05 \mathrm{eV}$. Good agreement with theoretical calculations of chemical shifts has been obtained ${ }^{[104]}$ so that an overall consistent set of data is available now that is presented in Table 1. The table reports data for a set of species compatible with the scenario sketched in the case of the $\mathrm{Cu}$ oxidation discussed above. The metal converts into a surface oxide and a sub-surface species that is not discriminated at this point. The surface oxide grows in a thicker precursor phase that is defective at low growth temperatures forming with increasing temperature a thinner film and finally produces $\mathrm{RuO}_{2}$ at potentials allowing bulk equilibration. The sensitivity of the $\mathrm{Ru} 3 \mathrm{~d}_{5 / 2}$ core level is high enough to resolve shifts due to differences in coordination. For example in the metallic state it resolves surface and second-layer sub-surface species. In the adsorbate and surface oxide species different co-ordination variants can be resolved. Defects in the low-temperature oxide and coordinatively undersaturated sites in the stoichiometric oxide are also distinguishable. It is possible that other systems could also be determined with similar resolution and with the confidence level provided by the extensive theoretical coverage. For the present purposes this system serves as excellent model case for metal-oxygen catalytic systems. The range of oxygen 1s binding energies is exactly the same as that found for the copper system except that the mono-valent $\mathrm{Cu}_{2} \mathrm{O}$ appears at the higher binding energy of 530.5 $\mathrm{eV}$ whereas the $\mathrm{CuO}$ phase is very close to the stoichiometric $\mathrm{RuO}_{2}$ system. The table also lists the oxygen binding energies of important adsorbate states relevant for the present discussion. They also agree quite well with literature data.

Figure 28 summarizes the key results of the CO oxidation study. The O 1s XPS data imply at first sight that adsorbed and sub-surface species prevail at low temperature whereas above $500 \mathrm{~K} \mathrm{Ru}$ oxide ${ }^{[92]}$, the proposed 'active phase', is growing. The evolution of the $\mathrm{Ru} 3 \mathrm{~d}_{5 / 2}$ core level shows that the sequence of events is more complex and that the precursor surface oxide $\mathrm{Ru}_{\mathrm{x}} \mathrm{O}_{\mathrm{y}}$ appears as intermediate. When these complex transformations are compared to the conversion curve, also recorded in-situ, it becomes apparent that the catalytic activity is insensitive to the phase transformations. This result is in agreement with the theoretical predictions ${ }^{[80]}$, indicating that a mixture of adsorbate phases will exhibit the highest activity. The maximum activity occurs in a region of the 
phase diagram where the surface energies of metal and oxide phases are comparable. In this area the interconversion of phases should be most facile and the chance of existence of metastable intermediates such as sub-surface oxygen species should be highest. The most active sites from a "transient oxide" become available as soon as the transformation sets in, i.e. as soon as the catalyst surface becomes metastable.

The explanation of the "structure insensitivity" of the reaction at intermediate pressures is also given in Figure 28. The inset in the top right panel shows a micrograph of the distribution of the surface oxide and $\mathrm{RuO}_{2}$ phases. A patchy nature is clearly seen. The perimeter sites of the two phases may also be the location of active sites that are different from those available below $430 \mathrm{~K}$, the temperature of the onset of the segregation of the sub-surface components into the precursor surface oxide. It has been shown ${ }^{[101,102]}$ that the pattern is not static but dynamic, due to the interplay between COcovered and oxygen-covered patches. The mutual feedback between sub-surface species and its strain-restructuring activity (see Figure 27) is the driver for the dynamics allowing for a fast redistribution of active sites over the surface and preventing the domination of any of the model phases prepared in single crystals under these reaction conditions. Only at temperatures above $600 \mathrm{~K}$ a stoichiometric $\mathrm{RuO}_{2}$ thick layer is produced, its formation coinciding with the deactivation of the catalyst. These observations are in excellent agreement with the atmospheric pressure data obtained on Ru nanoparticles ${ }^{[57,94]}$, which also predict that a metastable thin film of oxidic nature, rather than pure metal or $\mathrm{RuO}_{2}$, is the most active phase in this reaction.

The active sites should comprise electrophilic oxygen oxidizing adsorbed $\mathrm{CO}$. The theoretical modeling of the reaction ${ }^{[93]}$ revealed that counterintuitive to the structural model of $\mathrm{a} \mathrm{RuO}_{2}$ (110) surface, oxygen atoms adsorbed on under-coordinated $\mathrm{Ru}$ atoms contribute the most to the reactivity. A mechanism where $\mathrm{Ru}$ atoms would be the sites of $\mathrm{CO}$ adsorption in a Mars-van-Kreveln type reaction with bridging oxygen atoms was found, for microkinetic reasons, to be less relevant despite its favorable energetics. These theoretical findings translate into the chemistry of atomic oxygen in the following way: bridge oxygen is nucleophilic and thus less likely to react with $\mathrm{CO}$, which is a strong nucleophile when adsorbed on a metal. If however the metal is deprived of some of its electron density, as for example when covalently bound to oxygen, then certain reactivity 
can be expected, as electron donation of the adsorbed $\mathrm{CO}$ will exceed the back donation through the metal. The oxygen adsorbed on an undercoordinated metal site will be an electrophile, with its electrophilicity being the stronger the less electron density is located at the metal site. In summary, electronically diluted (partly oxidized) metal atoms resulting from sub-surface oxygen, combined with the strain they produce ${ }^{[56,99]}$ are the sites of high activity in $\mathrm{CO}$ oxidation. Thus oxygen adsorbed on pristine Ru is not very active (see low activity branch at low T in Figure 28) and transient or bulk oxides are also not highly active ${ }^{[103,105]}$ for CO oxidation as the active species are too nucleophilic. Undercoordinated metal atoms (rough, steps), with the additional strain distortion of their local electronic structure due to the sub-surface oxygen atoms, provide the most active state of Ru for CO oxidation. This state, existing according to the XPS data (Figure 28) between 450 and $600 \mathrm{~K}$, is however increasingly metastable. With increasing temperature it segregates into metal and surface oxide and eventually bulk oxide, which is the deactivating species in the system. The kinetics of the equilibration of the metastable active state is controlled by the nanostructuring of the Ru, the surface faceting ${ }^{[57]}$ modified by bulk-dissolved oxygen, its grain boundary structure and the chemical potential of oxygen moderated by the reducing species co-existing at the surface. The high-pressure XPD results identified the possible structures of the active phase and bring together all components of explanation provided by low-pressure experiments.

Supporting evidence for the relevance of surface roughness and the profound influence of sub-surface species is provided by the SEM images of a polycrystalline Ru catalyst shown in Figure 29. The material was pretreated in oxygen at $1200 \mathrm{~K}$ to obtain the sharply structured $\mathrm{RuO}_{2}$ seen in the top image. After $\mathrm{CO}$ oxidation at ambient pressure and at $488 \mathrm{~K}$ for $24 \mathrm{~h}$ the system was strongly deactivated and revealed a complete restructuring with a substantial decrease of particle size indicating a grain-boundary mediated destruction process of the starting material.

The results of the $\mathrm{CO}$ oxidation reaction predict that the methanol oxidation reaction would show a complementary behavior in structure-function relationship. This is because methanol oxidation requires nucleophilic oxygen as the active species. Since a fully oxygen-terminated $\mathrm{RuO}_{2}$ surface can be expect at substantial oxygen pressures, and since at low temperatures the adsorption of methoxy poisons the catalyst surface, it may be 
expected that the transient oxide state preceding deactivation of $\mathrm{CO}$ oxidation would be a good candidate for the active phase.

As this state is intermediate between oxide and metal, experiments were conducted in which polycrystalline $\mathrm{Ru}$ as well as $\mathrm{Ru}(0001)$ surfaces were first fully oxidized and then subjected to a $\mathrm{MeOH}$ - oxygen mixture. After this the substrates were fully reduced by sputtering and annealing to the pure metallic state and subject to the same reaction mixture. The conditions were: total pressure: $2.4 .10^{-1}$ mbar, $\mathrm{P}_{\mathrm{CH} 3 \mathrm{OH}} / \mathrm{P}_{\mathrm{O} 2}$ ratio $=1.5$ under which a high productivity for formaldehyde, a low combustion selectivity and no decomposition to $\mathrm{CO}$ and hydrogen were observed. It is thus adequate to use the production of formaldehyde as parameter for structure-function correlations. The key results are collected in Figure 30. The starting nature of the substrate was found to be unimportant, i.e., polycrystalline and single crystalline material behaved in very comparable manner. This excludes any strong influence of facet orientation under these high-pressure conditions. The active phase must be nanostructured and self-organized under the reaction conditions. The lower panels in Figure 30 show high-resolution spectra at selected temperatures during a temperature scan. The most striking observation is the evolution of all spectra into a unique set of data starting from clearly different chemical states and ending at exactly the same state being neither oxide nor metal. All observed peaks are assigned as shown in Table 1. A broadening of the Ru 3d spectrum towards a mixture of adsorbed and surface oxide with no bulk oxide component takes place. Bulk oxide would be the final state in the absence of methanol under these reaction conditions. The oxygen spectrum reveals adsorbed and dissolved oxygen species as well as the presence of methoxide from the beginning of reactivity. The oxide system undergoes reduction into a large fraction of metal species and with the same abundance of adsorbed and surface oxide as residues than present in the initially metallic system. The transition occurs through intermediates (the defective oxide and the surface oxide from Table 1) as can be seen from the poor resolution of the spectra at intermediate temperatures containing additional spectral weights than the initial and final state of the evolution. This behavior is reflected in the conversion data shown at the top of Figure 30. Above $500 \mathrm{~K}$ the performance is identical when the differing surface areas of the two samples are normalized out. At low temperature the oxide, with already partly reduced patches, is 
more active than the metal where at low temperatures the adsorbed electrophilic states that favor $\mathrm{CO}$ oxidation dominate.

The separation of oxygen species into electrophilic and nucleophilic varieties becomes possible through comparing the catalytic performance of the system as function of the intensity evolution of the combined non-oxidic contributions to the $\mathrm{Ru} 3 \mathrm{~d} 5 / 2$ spectrum shown also in Figure 30. The nucleophilicity of the oxygen in the transient oxidized state ( $\mathrm{O}$ sub) is controlled by the level of the chemical potential of oxygen as predicted in the scheme of Figure 28. If the ratio oxygen to methanol is changed to be more rich in oxygen then the selectivity changes to total combustion via observable formate without any change in the spectral positions of the peaks but with an increased abundance of the transient surface oxide to an extent where presumably a closed layer of this species and not a patchy co-existence with still bare metal is present. This is expected to have consequences on the evolution of strain and/or the roughening of the reactive surface. The quantification of these states is still in progress but even with nondestructive depth profiling it is a challenge to determine the fractional surface coverage of the surface oxide in the presence of sub-surface oxygen as the $O$ peaks have very similar binding energy.

The identification of the nature of the surface oxide on metallic Ru provides a basis for understanding the beneficial effect of "ruthenic acid" ${ }^{[58]}$, an oxi-hydrate used in Ru/C methanol oxidation catalysts in fuel cells. Such additives may act as oxygen reservoirs to maintain the adequate level of sub-surface oxygen that can segregate and thus stabilize the surface oxide that is subject to strong reductive stress at the reaction conditions in boiling methanol. It could be promising for addressing the selectivity issue in DMF devices if care would be taken not to fully reduce the Ru nanoparticles on carbon or other reducing supports.

\subsubsection{Copper in MeOH steam reforming}

The synthesis, oxidation and steam reforming of methanol are three important technical reactions carried out over $\mathrm{Cu}$ catalysts. They are related with each other via a common reaction intermediate, namely formate, that was found to play a crucial role in 
surface science experiments ${ }^{[48,78,88,106]}$. In comparative studies on different supports ${ }^{[71,}$

${ }^{72]}$ it was found that the redox properties of $\mathrm{Cu}$ play a critical role in steam reforming systems. This is due to its ability to switch from methanol decomposition that produces $\mathrm{CO}$, to direct oxidation, in contrast to the pathway of consecutive decomposition followed by $\mathrm{CO}$ oxidation. These findings correlate well with the previous discussion about the critical role of sub-surface oxygen made possible by the use of in-situ XPS studies. In the framework of the scheme in Fig. 27, it is obvious that no nucleophilic oxygen should be present in steam reforming to avoid total combustion. Electrophilic oxygen from water splitting may be useful to create formate and to burn $\mathrm{CO}$ to $\mathrm{CO}_{2}$. In an extensive mechanistic study summarizing the various concepts of steam reforming over $\mathrm{Cu}^{[107]}$ a reaction sequence was proposed, based on kinetic information, DRIFTS measurements and prior suggestions, that involve two types of active sites (SA, SB) that are nucleophilic and hold protons reversibly, and electrophilic holding C-O species coexisting next to each other on the active surface. Under the reaction conditions bulk copper metal cannot fulfill these requirements because it would not bind methanol or would be oxidized to higher valence states by intermediates forming strong bonds to the surface. One such intermediate is the formate species detected by DRIFTS in in-situ experiments. The fact that dissociative adsorption of water is kinetically not relevant points to the presence of metal sites capable of reversibly holding a proton by hydroxyl formation without forming a $\mathrm{Cu}$-hydroxide. In light of the in-situ XPS findings, it is plausible that the active site contains besides $\mathrm{Cu}$ atoms surface oxygen created by bulkdissolved oxygen, with the lattice strain preventing its relaxation into a strongly bound oxide or hydroxide. In the framework of scheme 27, the "in-surface" dissolved $\mathrm{O} \gamma$ species, in steady state equilibrium with oxygen adsorbed above and sub-surface oxygen (electrophilic), fulfills these conditions. The requirement of lattice strain is important because under steam reforming conditions the oxygen chemical potential is only moderate and surface segregation into surface oxides that would be quickly hydroxylated by the excess of dissociatively adsorbed water must be avoided. The proposed site would fulfill all requirements of the SA and SB sites since strained $\mathrm{Cu}$ is a candidate for reversible adsorption of oxygenates and the in-surface oxygen could hold protons by forming strongly polarized surface hydroxyl species. The active surface would thus be 
free of $\mathrm{Cu}$ oxides and $\mathrm{Cu}-\mathrm{Zn}$ hydroxide species, which are proxies for oxidized surface sites that block and inhibit catalytic action.

It is not possible to directly detect the small fraction of bulk-dissolved and in-surface oxygen, since under reaction conditions the nanostructured $\mathrm{Cu}-\mathrm{ZnO}$ catalyst is covered by $\mathrm{ZnO}$ (see figure 23). Rather, a bracketing approach has to be adopted excluding the presence of species that should not be present on the active surface. The in-situ C 1s XPS data revealed a drastic change from the precursor to the activated system and did not change any further during reaction. The high fractional $\mathrm{ZnO}$ surface coverage did not change either. Figure 31 indicates that in the calcined state, besides the expected carbon from unspecific hydrocarbon fragment residues around $285 \mathrm{eV}$ and carbonates at 290.3 $\mathrm{eV}$ (see also $\mathrm{CO}_{2}$ evolution in Figure 22), methoxy and formate species are present. This is a good indication for the activity of the $\mathrm{Cu}$ system where auto reduction from hydroxycarbonate precursors was sufficient to create strongly attached methoxy and formate species. It is not possible to determine the nature of the cation in these species, and it is quite possible that $\mathrm{Zn}$ accounts for a substantial fraction. The binding energy of methoxy $(286.3 \mathrm{eV})$ and of formate $(288.5 \mathrm{eV})$ agree well with those found of $\mathrm{Cu}$ single crystals ${ }^{[77]}$ when the binding energy scales are adjusted with respect to the unspecific carbon/hydrocarbon species.

It is clear from these data that under reaction conditions the activated catalyst contains a large amount of unspecific carbon/hydrocarbon species and a small amount of methoxy and formate, but no carbonate. This is a sign of the absence of Broensted basic surface hydroxides as the product $\mathrm{CO}_{2}$ would otherwise form an overlayer of carbonates as those in the catalyst precursor ${ }^{[50]}$. The presence of surface hydroxide compounds would be inhibiting the reaction if they were covering the active surface. These findings compare well with the literature where in methanol oxidation at lower pressures ${ }^{[77]} \mathrm{a}$ small coverage of the active surface with carbon-containing intermediates was found and with atmospheric pressure DRIFTS experiments where a similar coverage of methoxy and formate in a 2.5:1 ratio was detected. The comparison shows that for this type of reactions a pressure in the millibar range is adequate to represent "real" reaction conditions. 
Oxygen 1s XPS of the catalyst during a temperature-programmed experiment from $300 \mathrm{~K}$ to $523 \mathrm{~K}$ in steam reforming feed are displayed in Figure 32. The colored spectrum marks the onset of activity. The inset in Figure 33 indicates the thermal evolution of the hydrogen production. Two components of the oxygen peak are visible in the spectra. The binding energy range covered excludes the presence of any oxidic copper species, in agreement with the above speculation about the active site. The lower binding energy peak is assigned mostly to oxygen in $\mathrm{ZnO}$ and called "oxidic" peak. Electrophilic and sub-surface oxygen species on copper could also contribute to this peak. The higher binding energy peak is assigned to hydroxide and defect species in $\mathrm{ZnO}$. The oxygen contributions of methoxy and formate contribute to this peak as well as dissociatively adsorbed water. The correlations in Figure 33 agree well with the above discussion about the detrimental role of the hydroxide and the possibility that bulk-dissolved oxygen in $\mathrm{Cu}$ is the active phase of the catalyst. The onset of activity, marked by the red spectrum in Figure 32, clearly correlates with the beginning of the splitting in relative intensity. The removal from the $\mathrm{ZnO}$ surface of any form of adsorbate and hydroxide seems to be in line with the high activity of the catalyst. In agreement with the mechanistic kinetic study ${ }^{[107]}$ it is evident that a high coverage of dissociated water and/or methoxy/formate is detrimental for the evolution of activity and that the presence of activated water is of no relevance for the overall kinetics as it is reacting quickly with the organic species. A confirmation of the above peak assignment is obtained from the non-destructive depth profiling performed during reaction at $523 \mathrm{~K}$. The spectra in Figure 32 show that, as the kinetic energy increases (equivalent to an increase in information depth) the low binding energy peak, attributed to the $\mathrm{ZnO}$, increases while the higher binding energy peak, due to hydroxyls is to a large extent a surface-adsorbed species. The presence of some $\mathrm{ZnO}$ as thin film over $\mathrm{Cu}$ and the existence of hydroxyl species in the bulk of defective $\mathrm{ZnO}$ are the origin for a rather smooth dependence of relative intensities on increased information depth indicating the absence of a sharp interface between surface and bulk oxygen species.

From these data and the in-situ methanol oxidation experiments as well as from the high-pressure steam reforming kinetic experiments a consistent picture of the active phase emerges at the interface between a metallic copper and an oxidic $\mathrm{Zn}$ species. Any 
coverage with substantial amounts of hydrogen or carbon containing species seems detrimental, in agreement with the fast turnover of the methanol and water reactants. The synergistic interaction between $\mathrm{ZnO}$ and $\mathrm{Cu}$ could be the role of $\mathrm{ZnO}$ to stabilize the trace content of oxygen in $\mathrm{Cu}$ keeping it in a strained ${ }^{[56]}$ and surface-disordered state. It is not essential to postulate a spill-over function of the $\mathrm{ZnO}$ component. In the role of an oxygen regulating agent the defective $\mathrm{ZnO}$ form stabilizes the chemical potential of oxygen in the surface by providing oxygen in the reducing cycles of the catalyst life, and by absorbing oxygen emerging from the bulk reservoir that could lead to surface-oxide formation, in the more oxidizing cycles. This buffer action would also explain the long lifetime of such a delicate system. In the reaction environment of methanol and water, the $\mathrm{Cu} / \mathrm{ZnO}$ system may thermodynamically be in the form of a bulk metal and a stoichiometric oxide at high chemical potentials of oxygen, or in the form of a binary alloy at low chemical potentials of oxygen. The present experiments give rise to a surface structural model in which the two sites postulated in the literature ${ }^{[107]}$ could merge into a single active site. Such a site never considered before would contain oxygen-modified copper metal being stabilized by defective $\mathrm{ZnO}$. It is revealing to see that such a picture with, however, very different arguments and not supported by experimental direct evidence ${ }^{[54]}$ also emerged for the active site of a methanol synthesis catalyst that should, according to the initial hypothesis of this section, be structurally equivalent to the site required for methanol steam reforming.

\subsubsection{Automobile catalysis: coadsorption of $\mathrm{CO}$ and $\mathrm{NO}$ on $\mathrm{Rh}$}

In the last two examples of application of APPES we show noble metal catalysis experiments where the substrate does not experience the massive restructuring involved in formation of oxide films. In the first one we show how APPES was used to determine the nature of the sites occupied by $\mathrm{CO}$ and $\mathrm{NO}$ as they compete for adsorption on $\mathrm{Rh}(111)$, a model reaction for the reduction of $\mathrm{NO}$ and oxidation of $\mathrm{CO}$ in automobile exhaust catalysts ${ }^{[108]}$. Knowledge of the structure and site occupation of mixed CO and $\mathrm{NO}$ adsorbates is key to understanding the catalytic process. Studies of such mixed system were carried out using thermal-programmed desorption by Root et al. ${ }^{[109]}$ and by 
Witte using He scattering ${ }^{[10]}$. Using the $\mathrm{CO}$ and NO stretch mode frequencies in the IR spectra as indicators of the adsorption site, Permana et al. ${ }^{[111]}$ have shown that under catalytic reaction conditions NO occupies hollow sites while CO occupies top sites. Molecular resolution images have been obtained by Rider et al. using STM under catalytic pressure conditions (in the mbar range ${ }^{[112]}$. STM however could resolve only the molecules adsorbed on top sites, which account for $1 / 3$ of the total number. The other 2/3 consists of molecules adsorbed on hollow sites, which are invisible in the STM images. The authors of the STM study proposed a model for the molecular structure based on the analysis of exchange events observed with STM between the top CO and $\mathrm{NO}$ molecules. In this model as the partial pressure of $\mathrm{NO}$ increases in the $\mathrm{CO}+\mathrm{NO}$ gas mixture, $\mathrm{NO}$ displaces $\mathrm{CO}$ first in the hollow sites and later on the top sites. The experiments performed using APPES confirmed that assignment.

The experiments were performed at the ALS in beamline 9.3.2 by Requejo et al. ${ }^{[108]}$ using the first version of the APPES instrument. The Rh(111) crystal was cleaned by heating in $\mathrm{O}_{2}$ to remove carbonaceous contaminants followed by reduction of the oxide by reaction with $\mathrm{CO}$. The XPS experiments were performed in the pressure range between $10^{-3}$ to $650 \mathrm{~Pa}$. The incident photon energy was set at $450 \mathrm{eV}$ for $\mathrm{C} 1 \mathrm{~s}$ and 650 $\mathrm{eV}$ for $\mathrm{N} 1 \mathrm{~s}$ so that the emitted photoelectrons had comparable kinetic energies, close to $150 \mathrm{eV}$. The results are shown in figure 34. Spectrum labeled $100 \% \mathrm{CO}$ was obtained at room temperature in the presence of $20 \mathrm{~Pa}$ of pure $\mathrm{CO}$ gas. Under these conditions $\mathrm{CO}$ saturates the surface occupying the top sites and the hcp and fcc hollow sites of a $2 \times 2$ unit cell. The binding energies of in the $\mathrm{C} 1 \mathrm{~s}$ region clearly show the two species top $(\mathrm{T})$ and hollow $(\mathrm{H})$. After addition of $6.6 \mathrm{~Pa}$ of $\mathrm{NO}$ to the gas phase (27\% of the total pressure) the peak corresponding to $\mathrm{CO}$ on hollow sites has essentially disappeared. The $\mathrm{N} 1 \mathrm{~s}$ region of the spectrum shows that NO reaches close to its saturation coverage intensity under the CO-NO mixture. Displacement of the top CO species is a much slower process, observed by the small decrease of the intensity of its XPS peak. In contrast with the facile displacement of the hollow site CO species, the top site CO persisted and its complete removal could only be achieved by evacuation of the $\mathrm{CO}$ and after heating to $623 \mathrm{~K}$.

\subsubsection{Poisoning of $\mathrm{H}_{2}$ chemisorption by $\mathrm{CO}$ on $\mathrm{Pt}(111)$}


In the final example we show how APPES can reveal the surface coverage of species that block metal active sites. It opens the interesting possibility of monitoring the effect of the ensemble size of non-blocked catalyst atoms in the reactive adsorption and dissociation of reactant molecules. The reaction chosen by Montano et al. ${ }^{[113]}$ was a simple one: the dissociation of $\mathrm{H}_{2}$ on $\mathrm{Pt}(111)$, monitored by the yield of the exchange reaction with $\mathrm{D}_{2}$. This simple reaction is very important in the removal of $\mathrm{CO}$ from mixtures of $\mathrm{CO}$ and $\mathrm{H}_{2}$ and a crucial step in the water-gas shift and related reactions because $\mathrm{Pt}$ catalysts are poorly tolerant of $\mathrm{CO}$ poisoning in hydrogenation reactions. By comparing the HD yield and the $\mathrm{CO}$ coverage as a function of temperature and pressure the authors address the question of how many Pt sites need to be blocked by $\mathrm{CO}$ to effectively poison the dissociative adsorption of $\mathrm{H}_{2}$. The experiments were performed in two different instruments. One is an STM in a reaction chamber, which was used to monitor the structures formed on $\mathrm{Pt}(111)$ exposed to a mixture of $\mathrm{CO}, \mathrm{H}_{2}$, and $\mathrm{D}_{2}$. The results of the reactivity experiments performed in this chamber are shown in figure 35 . They show that the activation energy for $\mathrm{H}_{2}-\mathrm{D}_{2}$ exchange increased from $5.35 \mathrm{kcal} / \mathrm{mol}$, in the clean surface when no $\mathrm{CO}$ was present, to $17.44 \mathrm{kcal} / \mathrm{mol}$ when $0.7 \mathrm{~Pa}$ of $\mathrm{CO}$ were added to the $27 \mathrm{~Pa} \mathrm{H}_{2}+2.7 \mathrm{~Pa} \mathrm{D}_{2}$ mixture, clearly as a result of $\mathrm{CO}$ poisoning. Unfortunately STM could only reveal the static ordered structures of $\mathrm{CO}$ when it blocks the surface sites completely. The STM images revealed an incommensurate structure, similar to that reported ${ }^{[14]}$ in previous STM studies. As can be noticed in the schematic diagram of this structure, shown as an inset in figure 36, the adsorption sites are not exactly on top or on bridge, if the spacing and angle of the $\mathrm{CO}$ molecules are maintained rigid and equally spaced. Relaxations around these positions however could bring the adsorption sites closer to top and bridge as found in the commensurate $c(2 \times 4)$ structures $[115,116]$. During reaction conditions, the highly mobile CO vacancies present at the surface make the reaction possible but could not be detected by the STM due to the slow acquisition time of the images.

The authors studied the same reaction by APPES in beamline 11.0.2 of the ALS. After cleaning, the $\operatorname{Pt}(111)$ crystal was introduced in the high pressure reaction chamber, which was then backfilled with the same reaction mixture of $\mathrm{H}_{2}, \mathrm{D}_{2}$, and $\mathrm{CO}$ as in the 
previous experiments. The platinum $\mathrm{Pt} 4 \mathrm{f}, \mathrm{C} 1 \mathrm{~s}$, and $\mathrm{O} 1 \mathrm{~s}$ peaks were recorded at incident photon energies of 230, 450, and $690 \mathrm{eV}$, respectively, so that the photoelectron kinetic energies, and therefore the surface sensitivity, were the same. XPS spectra of the C1s peaks from adsorbed $\mathrm{CO}$ in equilibrium with gas at $298 \mathrm{~K}$ and a pressure of $27 \mathrm{~Pa} \mathrm{H}_{2}, 2.7$ $\mathrm{Pa} \mathrm{D}_{2}$, and 0.7 $\mathrm{Pa} \mathrm{CO}$ are shown in figure 36. Two XPS peaks can be resolved at 286.8 and $286.1 \mathrm{eV}$. In the O1s region the peaks were at 531.5 and $533.5 \mathrm{eV}$. Similar values for the $\mathrm{C} 1 \mathrm{~s}$ and $\mathrm{O} 1 \mathrm{~s}$ peaks were obtained in low-pressure studies at $200 \mathrm{~K}$ for a c $(4 \times 2) \mathrm{CO}$ structure on $\operatorname{Pt}(111)$ structure, although in that case the peaks were about $30 \%$ narrower. They were assigned to top site (286.8 and $533.5 \mathrm{eV})$ and bridge-site adsorption $(286.1 \mathrm{eV}$ and $531.5 \mathrm{eV}$ ). The incommensurability found in Pt could explain the broader XPS peaks measured in that case.

The XPS peaks were monitored as the surface temperature was increased from 298 to $480 \mathrm{~K}$ in the gas mixture. For quantitative analysis the $\mathrm{C} 1 \mathrm{~s}$ and $\mathrm{O} 1 \mathrm{~s}$ peaks areas were normalized to the initial saturation coverage of 0.6 ML of CO. Due to their larger splitting the O1s peaks were used. The resulting data are plotted in Figure 37. As the temperature is increased, the total coverage decreases from $0.6 \mathrm{ML}$ at $298 \mathrm{~K}$ to $0.47 \mathrm{ML}$ at $345 \mathrm{~K}$, followed by a plateau and a further decrease above $400 \mathrm{~K}$ to reach $0.34 \mathrm{ML}$ at $480 \mathrm{~K}$.

A $20 \%$ decrease of CO coverage occurs from 298 to $345 \mathrm{~K}$. In the reactivity experiments this corresponds to an increase in turnover frequency for HD production from below the detection limit to 0.03 molecule/site/s. While measurable, this value is 3 orders of magnitude smaller than in the absence of $\mathrm{CO}$ in the same temperature range, indicating that the reactivity does not scale proportionally to the area free of $\mathrm{CO}(0.2$ ML). If dissociative adsorption were the rate-limiting step, one likely explanation is that aggregates of several vacancy sites are needed for the reaction to occur. Such an effect has indeed been observed at low temperature for the dissociation of $\mathrm{H}_{2}$ on $\mathrm{Pd}(111)$, which requires ensembles of three or more free sites ${ }^{[117]}$. If a similar argument is valid for vacancies in a CO-covered surface, a coverage of $0.2 \mathrm{ML}$ vacancies would generate $\mathrm{n}$ vacancy sites in proportion to $0.2^{\mathrm{n}}$. The observed decay in reactivity of $10^{-3}$ relative to the clean surface would imply $\mathrm{n} \sim 4$. Another possible explanation is that CO simply limits the ability of $\mathrm{H}$ and $\mathrm{D}$ to diffuse across the surface. However, given the similarity in 
activation energy of the exchange reaction on the poisoned surface to the desorption energy of $\mathrm{CO}$ it appears that the creation of vacancy aggregates is indeed the rate-limiting step.

\section{Outlook}

The present review has highlighted the technique of elevated pressure photoemission or ambient pressure photoelectron spectroscopy. While the technique is still young and its potential has not yet fully developed, we have already shown that it can have a substantial impact in areas of environmental and atmospheric chemistry that involve liquid water and ice. We have shown its usefulness in the catalysis area for analysis of surface species, reactants and products during the reaction conditions, and throwing experimental light on the transition from surface-dominated reactions to sub-surfaceinduced chemistry. This transition represents a good deal of the "material gap" problem in model catalysis and is now directly accessible. Much more work has to be done to apply the method to insulating materials where the intense synchrotron beam causes substantial problems of charging and of beam modification.

With the advent of more instruments at various synchrotrons it is expected that other areas of material and nano-science will benefit from the methodical development. For such potential new areas the possibilities and limitations of the method outlined in this review will also apply. When novel phenomena are being discovered care should be taken not to be misled by the enormous sensitivity of the technique against spurious contamination, beam damage and differential charging that can complicate the interpretation of the data. The enormous qualitative new insight becoming accessible through ambient pressure photoemission should, however, under all circumstances outweigh the difficulties in ascertaining the integrity of the results. 


\section{Acknowledgements}

M.S. was supported by the Director, Office of Energy Research, Office of Basic Energy Sciences, Materials Sciences Division, of the U.S. Department of Energy under Contract No. DE-AC02-05CH11231. R.S. acknowledges the MPG. The first phase of instrument development was made possible by an LDRD grant from the LBNL. The second generation instrument benefited from a fruitful collaboration between our institutions and the team of SPECS. The authors gratefully acknowledge all those who have contributed to the experiments described. We are grateful to our teams for implementing the two instruments. Without the contributions of F. Ogletree, H. Bluhm, E. Hebenstreit, F. Requejo, A. Knop-Gericke and M. Haevecker this project would not exist. We thank our synchrotron facilities ALS and BESSY and their staff for their longstanding support.

\section{References}

[1] P. B. Rasmussen, B. L. M. Hendricksen, H. Zeiljlemaker, H. G. Ficke, J. W. M. Frenken, Review of Scientific Instruments 1998, 69, 3879.

[2] E. Laegsgaard, L. Österlund, P. Thostrup, I. Stensgaard, F. Besenbacher, Review of Scientific Instruments 2002, 72, 3537.

[3] J. F. O`Hanlon, A User Guide to Vacuum Technology, John Wiley and Sons, 2003.

[4] R. Boschi, E. Clar, W. Schmidt, Journal of Chemical Physics 1974, 60, 44064418.

[5] K. Siegbahn, C. Nordling, G. Johannson, J. Hedman, P. F. Heden, K. Hamrin, U. Gelius, T. Bergmark, L. O. Werme, R. Manne, Y. Baer, ESCA Applied to Free Molecules, North Holland, Amsterdam, London, 1969.

[6] J. J. Pireaux, S. Svensson, E. Basilier, P. A. Malmqvist, U. Gelius, R. Gaudano, K. Siegbahn, Physical Review A 1976, 14, 2133-2145.

[7] B. Lindberg, L. Asplund, H. Fellnerfeldegg, P. Kelfve, H. Siegbahn, K. Siegbahn, Chemical Physics Letters 1976, 39, 8-10.

[8] H. Siegbahn, K. Siegbahn, Journal of Electron Spectroscopy and Related Phenomena 1973, 2, 319 - 325.

[9] H. Siegbahn, L. Asplund, P. Kelfve, K. Hamrin, L. Karlsson, K. Siegbahn, Journal of Electron Spectroscopy and Related Phenomena 1974, 5, 1059-1079. 
[10] H. Fellnerfeldegg, U. Gelius, B. Wannberg, A. G. Nilsson, E. Basilier, K. Siegbahn, Journal of Electron Spectroscopy and Related Phenomena 1974, 5, 643-689.

[11] R. Moberg, F. Bökman, O. Bohman, H. O. G. Siegbahn, J. Chem. Phys. 1991, 94, 5226-5232.

[12] R. W. Joyner, M. W. Roberts, K. Yates, Surface Science 1979, 87, 501-509.

[13] R. W. Joyner, M. W. Roberts, Chemical Physics Letters 1979, 60, 459-462.

[14] M. Grunze, D. J. Dwyer, M. Nassir, Y. Tsai, Acs Symposium Series 1992, 482, 169-182.

[15] D. F. Ogletree, H. Bluhm, G. Lebedev, C. S. Fadley, Z. Hussain, M. Salmeron, Review of Scientific Instruments 2002, 73, 3872-3877.

[16] R. Denecke, M. Kinne, C. M. Whelan, H. P. Steinruck, Surface Review and Letters 2002, 9, 797-801.

[17] M. A. Kelly, M. L. Shek, P. Pianetta, T. M. Gur, M. R. Beasley, Journal of Vacuum Science \& Technology a-Vacuum Surfaces and Films 2001, 19, 2127 2133.

[18] T. Tyliszczak, T. Warwick, A. L. D. Kilcoyne, S. Farka, D. K. Shuh, T. H. Yoon, G. E. Brown Jr, S. Andrews, Y. Acreman, in Synchrotron Radiation Instrumentation, Vol. 705, American Institute of Physics, 2004, p. 1356.

[19] J. Stöhr, NEXAFS Spectroscopy, Springer, Berlin, 1992.

[20] J. J. Yeh, I. Lindau, Atomic Data Nuclear Data Tables 1985, 32, 1.

[21] G. Moretti, Zeolites 1994, 14, 469-475.

[22] H. Shimada, N. Matsubayashi, M. Imamura, T. Sato, A. Nishijima, Catalysis Letters 1996, 39, 125-128.

[23] W. Grunert, R. Schlogl, H. G. Karge, Journal of Physical Chemistry 1993, 97, 8638-8645.

[24] H. Shimada, K. Sato, N. Matsubayashi, M. Imamura, T. Saito, K. Furuya, Applied Surface Science 1999, 145, 21-25.

[25] H. Bluhm, D. F. Ogletree, C. S. Fadley, Z. Hussain, M. Salmeron, J Phys. Cond. Matter 2002, 14, L227.

[26] S. Myneni, Y. Luo, L. A. Naslund, M. Cavalleri, L. Ojamae, H. Ogasawara, A. Pelmenschikov, P. Wernet, P. Vaterlein, C. Heske, Z. Hussain, L. G. M. Pettersson, A. Nilsson, Journal of Physics-Condensed Matter 2002, 14, L213L219.

[27] E. M. Knipping, M. J. Lakin, K. L. Foster, P. Jungwirth, D. J. Tobias, R. B. Gerber, D. Dabdub, B. J. Finlayson-Pitts, Science 2000, 288, 301-306.

[28] J. H. Hu, Q. Shi, P. Davidovits, D. R. Worsnop, M. S. Zahniser, C. E. Kolb, Journal of Physical Chemistry 1995, 99, 8768-8776.

[29] P. Jungwirth, D. J. Tobias, Journal of Physical Chemistry B 2002, 106, 63616373.

[30] S. W. Hunt, M. Roeselova, W. Wang, L. M. Wingen, E. M. Knipping, D. J. Tobias, D. Dabdub, B. J. Finlayson-Pitts, Journal of Physical Chemistry A 2004, 108, 11559-11572.

[31] S. Ghosal, J. C. Hemminger, H. Bluhm, B. S. Mun, E. L. D. Hebenstreit, G. Ketteler, D. F. Ogletree, F. G. Requejo, M. Salmeron, Science 2005, 307, 563566. 
[32] S. Ghosal, A. Verdaguer, J. C. Hemminger, M. Salmeron, Journal of Physical Chemistry A 2005, 109, 4744-4749.

[33] G. Ketteler, S. Yamamoto, H. Bluhm, K. Andersson, D. E. Starr, D. F. Ogletree, H. Ogasawara, A. Nilsson, M. Salmeron, Journal of Physical Chemistry C 2007, 111, 8278-8282.

[34] A. Verdaguer, C. Weis, G. Oncins, G. Ketteler, H. Bluhm, M. Salmeron, Langmuir 2007, 23, 9699-9703.

[35] K. Reuter, C. Stampfl, M. V. Ganduglia-Pirovano, M. Scheffler, Chemical Physics Letters 2002, 352, 311-317.

[36] K. Reuter, M. Scheffler, Applied Physics a-Materials Science \& Processing 2004, 78, 793-798.

[37] E. Lundgren, J. Gustafson, A. Mikkelsen, J. N. Andersen, A. Stierle, H. Dosch, M. Todorova, J. Rogal, K. Reuter, M. Scheffler, Physical Review Letters 2004, 92.

[38] G. Ketteler, D. F. Ogletree, H. Bluhm, H. J. Liu, E. L. D. Hebenstreit, M. Salmeron, Journal of the American Chemical Society 2005, 127, 18269-18273.

[39] E. Lundgren, G. Kresse, C. Klein, M. Borg, J. N. Andersen, M. De Santis, Y. Gauthier, C. Konvicka, M. Schmid, P. Varga, Physical Review Letters 2002, 88.

[40] M. Todorova, W. X. Li, M. V. Ganduglia-Pirovano, C. Stampfl, K. Reuter, M. Scheffler, Physical Review Letters 2002, 89.

[41] I. Barin, Thermochemical Data, VCH, Weinheim, 1992.

[42] T. Schedel-Niedrig, T. Neisius, N. Bottger, E. Kitzelmann, G. Weinberg, D. Demuth, R. Schlogl, Physical Chemistry Chemical Physics 2000, 2, 2407-2417.

[43] G. W. Zhou, L. Wang, J. C. Yang, Journal of Applied Physics 2005, 97.

[44] T. Kangas, K. Laasonen, A. Puisto, H. Pitkanen, M. Alatalo, Surface Science 2005, 584, 62-69.

[45] T. Kravchuk, A. Hoffman, Surface Science 2007, 601, 87-94.

[46] V. I. Bukhtiyarov, M. Havecker, V. V. Kaichev, A. Knop-Gericke, R. W. Mayer, R. Schlogl, Physical Review B 2003, 67.

[47] V. I. Bukhtiyarov, A. I. Nizovskii, H. Bluhm, M. Havecker, E. Kleimenov, A. Knop-Gericke, R. Schlogl, Journal of Catalysis 2006, 238, 260-269.

[48] M. Bowker, R. A. Hadden, H. Houghton, J. N. K. Hyland, K. C. Waugh, Journal of Catalysis 1988, 109, 263-273.

[49] G. C. Chinchen, M. S. Spencer, K. C. Waugh, D. A. Whan, Journal of the Chemical Society-Faraday Transactions I 1987, 83, 2193-2212.

[50] M. M. Gunter, T. Ressler, B. Bems, C. Buscher, T. Genger, O. Hinrichsen, M. Muhler, R. Schlogl, Catalysis Letters 2001, 71, 37-44.

[51] B. Bems, M. Schur, A. Dassenoy, H. Junkes, D. Herein, R. Schlogl, Chemistry-a European Journal 2003, 9, 2039-2052.

[52] P. L. Hansen, J. B. Wagner, S. Helveg, J. R. Rostrup-Nielsen, B. S. Clausen, H. Topsoe, Science 2002, 295, 2053-2055.

[53] J. D. Grunwaldt, A. M. Molenbroek, N. Y. Topsoe, H. Topsoe, B. S. Clausen, Journal of Catalysis 2000, 194, 452-460.

[54] M. S. Spencer, Catalysis Letters 1999, 60, 45-49. 
[55] T. Ressler, B. L. Kniep, I. Kasatkin, R. Schlogl, Angewandte ChemieInternational Edition 2005, 44, 4704-4707.

[56] F. Girgsdies, T. Ressler, U. Wild, T. Wubben, T. J. Balk, G. Dehm, L. Zhou, S. Gunther, E. Arzt, R. Imbihl, R. Schlogl, Catalysis Letters 2005, 102, 91-97.

[57] J. Assmann, V. Narkhede, L. Khodeir, E. Loffler, O. Hinrichsen, A. Birkner, H. Over, M. Muhler, Journal of Physical Chemistry B 2004, 108, 14634-14642.

[58] W. Sugimoto, T. Saida, Y. Takasu, Electrochemistry Communications 2006, 8, 411-415.

[59] Y. D. Kim, A. P. Seitsonen, S. Wendt, J. Wang, C. Fan, K. Jacobi, H. Over, G. Ertl, Journal of Physical Chemistry B 2001, 105, 3752-3758.

[60] H. Over, A. P. Seitsonen, Science 2002, 297, 2003-+.

[61] W. Unterberger, B. Jenewein, B. Klotzer, S. Penner, W. Reichl, G. Rupprechter, D. Wang, R. Schlogl, K. Hayek, Reaction Kinetics and Catalysis Letters 2006, 87, 215-234.

[62] S. Penner, W. Di, B. Jenewein, H. Gabasch, B. Klotzer, A. Knop-Gericke, R. Schlogl, K. Hayek, Journal of Chemical Physics 2006, 125.

[63] H. Gabasch, W. Unterberger, K. Hayek, B. Klotzer, E. Kleimenov, D. Teschner, S. Zafeiratos, M. Havecker, A. Knop-Gericke, R. Schlogl, J. Y. Han, F. H. Ribeiro, B. Aszalos-Kiss, T. Curtin, D. Zemlyanov, Surface Science 2006, 600, 2980-2989.

[64] D. Zemlyanov, B. Aszalos-Kiss, E. Kleimenov, D. Teschner, S. Zafeiratos, M. Havecker, A. Knop-Gericke, R. Schlogl, H. Gabasch, W. Unterberger, K. Hayek, B. Koltzer, Surface Science 2006, 600, 983-994.

[65] M. M. Gunter, B. Bems, R. Schlogl, T. Ressler, Journal of Synchrotron Radiation 2001, 8, 619-621.

[66] B. L. Kniep, T. Ressler, A. Rabis, F. Girgsdies, M. Baenitz, F. Steglich, R. Schlogl, Angewandte Chemie-International Edition 2004, 43, 112-115.

[67] I. Bottger, T. Schedel-Niedrig, O. Timpe, R. Gottschall, M. Havecker, T. Ressler, R. Schlogl, Chemistry-a European Journal 2000, 6, 1870-1876.

[68] M. S. Spencer, Catalysis Today 1992, 12, 453-464.

[69] H. Ludwiczek, A. Preisinger, A. Fischer, R. Hosemann, A. Schînfeld, W. Vogel, J.Catal. 1978, 51, 326-337.

[70] W. S. Borghard, M. Boudart, J.Catal. 1983, 80, 194-206.

[71] J. Agrell, M. Boutonnet, I. Melian-Cabrera, J. L. G. Fierro, Applied Catalysis a-General 2003, 253, 201-211.

[72] J. Agrell, H. Birgersson, M. Boutonnet, I. Melian-Cabrera, R. M. Navarro, J. L. G. Fierro, Journal of Catalysis 2003, 219, 389-403.

[73] R. N. d'Alnoncourt, X. Xia, J. Strunk, E. Loffler, O. Hinrichsen, M. Muhler, Physical Chemistry Chemical Physics 2006, 8, 1525-1538.

[74] M. Muhler, E. Tornqvist, L. P. Nielsen, B. S. Clausen, H. Topsoe, Catalysis Letters 1994, 25, 1-10.

[75] V. Bolis, B. Fubini, E. Giamello, A. Reller, Journal of the Chemical SocietyFaraday Transactions 1989, 85, 855-867.

[76] I. Kasatkin, B. Kniep, T. Ressler, Physical Chemistry Chemical Physics 2007, 9, 878-883. 
[77] S. Gunther, L. Zhou, M. Havecker, A. Knop-Gericke, E. Kleimenov, R. Schlogl, R. Imbihl, Journal of Chemical Physics 2006, 125.

[78] H. Werner, D. Herein, G. Schulz, U. Wild, R. Schlogl, Catalysis Letters 1997, 49, 109-119.

[79] K. Reuter, M. Scheffler, Physical Review B 2003, 68.

[80] K. Reuter, M. Scheffler, Physical Review Letters 2003, 90.

[81] H. Bluhm, M. Havecker, A. Knop-Gericke, E. Kleimenov, R. Schlogl, D. Teschner, V. I. Bukhtiyarov, D. F. Ogletree, M. Salmeron, Journal of Physical Chemistry B 2004, 108, 14340-14347.

[82] A. Knop-Gericke, M. Havecker, T. Schedel-Niedrig, R. Schlogl, Topics in Catalysis 2001, 15, 27-34.

[83] T. Schedel-Niedrig, M. Haavecker, A. Knop-Gericke, R. Schlogl, Physical Chemistry Chemical Physics 2000, 2, 3473-3481.

[84] A. Knop-Gericke, M. Havecker, T. Schedel-Niedrig, R. Schlogl, Catalysis Letters 2000, 66, 215-220.

[85] R. K. Grasselli, Topics in Catalysis 2002, 21, 79-88.

[86] A. F. Carley, P. R. Davies, M. W. Roberts, Philosophical Transactions of the Royal Society a-Mathematical Physical and Engineering Sciences 2005, 363, 829-846.

[87] A. F. Carley, P. R. Davies, M. W. Roberts, Catalysis Letters 2002, 80, 25-34.

[88] A. F. Carley, A. W. Owens, M. K. Rajumon, M. W. Roberts, S. D. Jackson, Catalysis Letters 1996, 37, 79-87.

[89] A. F. Carley, P. R. Davies, G. U. Kulkarni, M. W. Roberts, Catalysis Letters 1999, 58, 93-97.

[90] I. E. Wachs, R. J. Madix, Surface Science 1978, 76, 531-558.

[91] T. Zambelli, J. Wintterlin, J. Trost, G. Ertl, Science 1996, 273, 1688-1690.

[92] H. Over, Y. D. Kim, A. P. Seitsonen, S. Wendt, E. Lundgren, M. Schmid, P. Varga, A. Morgante, G. Ertl, Science 2000, 287, 1474-1476.

[93] K. Reuter, M. Scheffler, Physical Review B 2006, 73.

[94] H. Over, M. Muhler, Progress in Surface Science 2003, 72, 3-17.

[95] K. Reuter, M. Scheffler, Physical Review B 2002, 65.

[96] A. Bottcher, H. Conrad, H. Niehus, Journal of Chemical Physics 2000, 112, 4779-4787.

[97] A. Bottcher, H. Niehus, Journal of Chemical Physics 1999, 110, 3186-3195.

[98] A. Bottcher, H. Niehus, Physical Review B 1999, 60, 14396-14404.

[99] A. Bottcher, H. Niehus, Physica Status Solidi a-Applied Research 1999, 173, 101-107.

[100] G. Rotaris, A. Baraldi, G. Comelli, M. Kiskinova, R. Rosei, Surface Science 1996, 359, 1-9.

[101] R. Blume, M. Havecker, S. Zafeiratos, D. Teschner, E. Kleimenov, A. KnopGericke, R. Schlogl, A. Barinov, P. Dudin, M. Kiskinova, Journal of Catalysis 2006, 239, 354-361.

[102] R. Blume, H. Niehus, H. Conrad, A. Bottcher, L. Aballe, L. Gregoratti, A. Barinov, M. Kiskinova, Journal of Physical Chemistry B 2005, 109, 1405214058. 
[103] A. Bottcher, U. Starke, H. Conrad, R. Blume, H. Niehus, L. Gregoratti, B. Kaulich, A. Barinov, M. Kiskinova, Journal of Chemical Physics 2002, 117, 8104-8109.

[104] S. Lizzit, A. Baraldi, A. Groso, K. Reuter, M. V. Ganduglia-Pirovano, C. Stampl, M. Scheffler, M. Stichler, C. Keller, W. Wurth, D. Menzel, Physical Review B 2001, 6320.

[105] A. Bottcher, H. Conrad, H. Niehus, Surface Science 2000, 452, 125-132.

[106] T. S. Askgaard, J. K. Norskov, C. V. Ovesen, P. Stoltze, Journal of Catalysis 1995, 156, 229-242.

[107] B. Frank, F. C. Jentoft, H. Soerijanto, J. Krohnert, R. Schlogl, R. Schomacker, Journal of Catalysis 2007, 246, 177-192.

[108] F. G. Requejo, E. L. D. Hebenstreit, D. F. Ogletree, M. Salmeron, Journal of Catalysis 2004, 226, 83-87.

[109] T. W. Root, G. B. Fisher, L. D. Schmidt, Journal of Chemical Physics 1986, $85,4687-4695$.

[110] G. Witte, Journal of Chemical Physics 2001, 115, 2757-2767.

[111] H. Permana, K. Y. S. Ng, C. H. F. Peden, S. J. Schmieg, D. K. Lambert, D. N. Belton, Catalysis Letters 1997, 47, 5-15.

[112] K. B. Rider, K. S. Hwang, M. Salmeron, G. A. Somorjai, Journal of the American Chemical Society 2002, 124, 5588-5593.

[113] M. Montano, K. Bratlie, M. Salmeron, G. A. Somorjai, Journal of the American Chemical Society 2006, 128, 13229-13234.

[114] S. R. Longwitz, J. Schnadt, E. K. Vestergaard, R. T. Vang, E. Laegsgaard, I. Stensgaard, H. Brune, F. Besenbacher, Journal of Physical Chemistry B 2004, $108,14497-14502$.

[115] M. Kinne, T. Fuhrmann, C. M. Whelan, J. F. Zhu, J. Pantforder, M. Probst, G. Held, R. Denecke, H. P. Steinruck, Journal of Chemical Physics 2002, 117, 10852-10859.

[116] O. Bjorneholm, A. Nilsson, H. Tillborg, P. Bennich, A. Sandell, B. Hernnas, C. Puglia, N. Martensson, Surface Science 1994, 315, L983-L989.

[117] T. Mitsui, M. K. Rose, E. Fomin, D. F. Ogletree, M. Salmeron, Nature 2003, 422, 705-707. 